\title{
Sémiotique et onomastique dans Le Roi des Aulnes de Michel Tournier
}

\section{Nicolaas van der Toorn ${ }^{1}$ (D)}

Published online: 4 October 2018

(C) The Author(s) 2018

\begin{abstract}
This article addresses the ubiquitous presence of semiotics in Tournier's Le Roi des Aulnes. First, the different categories of signs are investigated, including the signs' influence on the protagonist Tiffauges, who reports on this influence in his journal. By doing so, Tiffauges involves the diary's narratee, and, indirectly, the novel's implied reader, into his struggling with signs and signification. Secondly, this article focusses upon one particular semiotic category, namely onomastics. This category, despite its omnipresence in the novel, has been underresearched until now. This article proposes a detailed analysis of the novel's onomastics in all its anagrammatical richness and ambiguity.
\end{abstract}

Keywords Le Roi des Aulnes · Signs · Names · Anagram · Wordplay · Inversion

Tout est signe. Mais il faut une lumière ou un cri éclatants pour percer notre myopie ou notre surdité. (MTR 193)

\section{Introduction}

Paru en septembre 1970 et obtenant le prix Goncourt à l'unanimité le mois suivant, Le Roi des Aulnes est le deuxième roman de Michel Tournier après Vendredi ou les Limbes du Pacifique. Garagiste à la Porte-des-Ternes à Paris, le protagoniste appelé Abel Tiffauges, droitier, constate accidentellement qu'il arrive aussi à écrire de la main gauche: il intitule son journal par conséquent Écrits sinistres. Ce géant myope de $110 \mathrm{~kg}$ se découvre de plus en plus friand de viande crue et de lait $(2 \mathrm{~kg}$ et 51 par jour), ce qui confirme sa vocation ogresse qui se manifeste également dans son

Michel Tournier, Romans, suivis de Le Vent Paraclet. Toute pagination du présent chapitre se rapportant à cette édition de la Bibliothèque de la Pléiade est précédée de l'abréviation MTR.

Nicolaas van der Toorn nvandertoorn@ziggo.nl

1 Center of the Arts in Society, Leiden University, Postbus 9515, 2300 RA Leiden, The Netherlands 
amour des enfants. Accusé de viol, Tiffauges est sauvé par la mobilisation en septembre 1939. Prisonnier de guerre, il est envoyé à Rominten, la réserve de chasse de Goering, puis en Prusse-Orientale où, accompagné de son cheval géant Barbe-Bleue, il écume la campagne à la recherche de garçons destinés à la formation d'officier SS dans l'internat de Kaltenborn.

\section{L'interprétation des signes}

Traditionnel en apparence, Le Roi des Aulnes est un roman plein de signes écrit par un auteur qui s'est plu à créer un personnage qui a du mal à voir, entendre et comprendre ces signes. Pour David Bevan « l'apogée sémiotique de l'œuvre de Tournier est Le Roi des Aulnes, où le signe est à tout moment souverain »(1986, pp. 67-68). Abel Tiffauges se plaint des difficultés qu'il rencontre à percevoir les signes, notamment par sa myopie, et il est obsédé par leur interprétation. Physique mais aussi mentale, cette myopie, appelée également cécité par lui, est surtout tournée vers l'extérieur, vers la réception des signes, car dès la première page du roman Tiffauges se montre extrêmement lucide et conscient de son moi et de ses origines immémoriales:

Je crois aussi que je suis issu de la nuit des temps. [...]. Quand la terre n'était encore qu'une boule de feu tournoyant dans un ciel d'hélium, l'âme qui la faisait flamber, qui la faisait tourner, c'était la mienne. Et d'ailleurs l'antiquité vertigineuse de mes origines suffit à expliquer mon pouvoir surnaturel [...]. (MTR 191)

Vraies ou plutôt fausses, cette conscience et cette connaissance de soi-même ( «je ne suis pas fou ${ }^{* 1}$ ) amènent Tiffauges à statuer du « sérieux total » de son journal. Ce garagiste explique et justifie les raisons de ses Écrits sinistres $*$ : « je compte en partie sur ce journal pour échapper à ce garage, aux médiocres préoccupations qui m'y retiennent, et en un certain sens à moi-même » (MTR 192). Il exige de son narrataire ${ }^{2}$ qu'il prenne son écriture au « sérieux », et « l'avenir aura pour fonction essentielle de démontrer - ou plus exactement d'illustrer - le sérieux ${ }^{*}$ des lignes qui précèdent ». D'emblée Tiffauges s'y présente précisément en ce qui le caractérise le plus: comme « ogre », « monstre féerique ». L'écriture de son journal correspond à cette nécessité sinon cette condamnation de s'exposer, de s'exhiber conformément à l'étymologie établie par Tiffauges des mots « monstrer »* et « monstrer » ${ }^{* 3}$. La constatation que « les monstres ne se reproduisent pas» (MTR 192) annonce les passages où il est question de la sexualité de Tiffauges, qui fait l'amour « comme

\footnotetext{
1 Tous les mots suivis d'un astérisque sont en italiques dans le texte.

2 Voir cependant note 10.

3 On peut s'étonner pourquoi Tiffauges ne parle pas de l'étymologie du mot ogre. Arlette Bouloumié (1988, pp. 89-91) et (2013, p. 340), en expose les différentes possibilités étymologiques. Voir aussi Platten (1999b, p. 89). Si « ogre » provient de « augure », Tiffauges est bien chargé d'observer des signes pour en tirer des présages et déterminer sa ligne de conduite en conséquence. La combinaison avec « Orcus », dieu de la mort qui règne sur l'enfer, complète le tableau ogresque.
} 
un serin » (MTR 195) et qui a le sexe, à l'âge de vingt ans, « d'enfant impubère », diagnostiqué microgénitomorphe* par un médecin local (MTR 249). En décrivant cette recherche étymologique réalisée, Tiffauges montre encore combien il est attentif aux signes, en l'occurrence aux mots et à leurs souches, attention en profondeur s'il en est ! D'emblée le lecteur est invité, sinon contraint, à suivre Tiffauges sur le chemin de l'étymologie, ${ }^{4}$ piste qui remonte dans le temps pour établir l'origine des mots. Tiffauges se dit lui aussi « issu de la nuit des temps » et parle de « l'antiquité vertigineuse de [s]es origines » (MTR 191). Il est établi un parallélisme entre la naissance de Tiffauges et celle des mots, souches qui sont situées à une époque immémoriale. Avec l'apocalypse imminente à la fin du roman, Tiffauges se dote ainsi d'une certaine intemporalité, caractéristique qu'il partage avec Nestor, son camarade du collège Saint-Christophe (MTR 205).

Le passage cité plus haut montre également le caractère franchement invraisemblable de ce journal réfléchi et hautement intellectuel tenu par un garagiste qui indique qu' " [à] la fin de ma seconde, il était clair que je ne passerais pas mon baccalauréat » (MTR 248). Dès les premières lignes du roman cette invraisemblance ${ }^{5}$ se confirme également au niveau du style soigné et travaillé du journal. C'est comme si, à son insu au départ, le lecteur était traîné par Tiffauges narrateur dans la zone d'ombre du caractère exceptionnel dont il tient à le convaincre.

Cette obsession des signes date de ses années d'initiation au collège Saint-Christophe où il « n' [a] cessé d'observer des hiéroglyphes tracés sur [s]on chemin ou d'entendre des paroles confuses ${ }^{6}$ murmurées à mes oreilles, sans rien comprendre, [...] mais aussi [...] la preuve réitérée que le ciel n'est pas vide. Or cette lumière [...] n'a pas fini d'éclairer ma route » (MTR 193). L'interprétation des signes détermine la conduite, la vie de Tiffauges. « [C]ette lumière », c'est d'abord la découverte récente par Tiffauges de sa capacité d'écrire de la main gauche. Perçue « avec un frisson sacré » (MTR 216), cette révélation est considérée par Tiffauges comme une rupture, qui s'ajoute à celle, tout aussi récente, avec la femme de sa vie Rachel. À la page MTR 247 Tiffauges écrit: « J'avais posé sur ma face le masque d'innocence dont je ne me suis pas départi depuis, mais que la rupture de Rachel, la découverte de l'écriture sinistre et quelques autres signes ${ }^{7}$ font curieusement trembler ». Avec son journal appelé « Écrits sinistres* » Tiffauges joue d'une manière évidente sur le double sens du mot sinistre, ambiguité qui est ainsi à la base du roman, ou tout au moins de la première partie du roman composée par le journal tenu par Tiffauges. L'écriture de la main gauche, donc sinistre au sens étymologique, est réservée aussi à la consignation de tous les sinistres rencontrés et (prétendument) provoqués par Tiffauges, qui la considère comme sincère par opposition à son « écriture adroite* » qui est feinte:

\footnotetext{
${ }^{4}$ Nous verrons plus loin que l'étymologie joue également un rôle important dans l'onomastique.

5 Plusieurs critiques ont relevé in extenso cette problématique de la vraisemblance et de certains anachronismes dans l'œuvre de Tournier. Citons Korthals Altes (1992, p. 157), Koopman-Thurlings (1991, pp. 279-293) et (1995), Vray (1997, pp. 395-398), Platten (1999b, pp. 177-185).

${ }^{6}$ Référence aux « confuses paroles » du deuxième vers de Correspondances (Les Fleurs du Mal), Charles Baudelaire.

7 Il ne précise pas de quels signes il s'agit.
} 
Je suis ainsi pourvu de deux écritures, l'une adroite ${ }^{*}$, aimable, sociale, commerciale, reflétant le personnage masqué que je feins d'être aux yeux de la société, l'autre sinistre ${ }^{*}$, déformée par toutes les gaucheries ${ }^{*}$ du génie, pleine d'éclairs et de cris, habitée en un mot par l'esprit de Nestor. (MTR 216-217)

Ambiguïté donc du mot sinistre, sur laquelle viennent se greffer des gaucheries* inhérentes au génie. Ambiguité aussi de l'adjectif adroite: l'écriture dextre, au sens propre donc de la main droite, se définit en effet au sens figuré par l'adresse imposée par l'adaptation sociale. C'est dans cette seule écriture sinistre que se trouvent « éclairs » et « cris » ou « lumière » et « cri » (MTR 193) permettant le déchiffrement des signes. C'est encore la découverte de cette écriture de la main gauche qui marque «la première émergence du nouveau Tiffauges, qui écrit [...] des choses neuves avec des mots que n'aurait pas trouvés à coup sûr mon écriture adroite ${ }^{8}$ » (MTR 248).

\section{De quels signes s'agit-il?}

Quand Tiffauges parle des signes, il leur confère dès le départ une double expression: auditive (« cri(s) ») ainsi que visuelle (« éclairs », « lumière »). Mais l'objectif recherché par Tiffauges avec son journal est aussi et avant tout de connaître mieux la nature des signes, recherche pour laquelle il revient à son enfance dans le collège Saint-Christophe avec son protecteur et alter ego Nestor dont « l'esprit habit[e] [son] écriture sinistre*». « Être monstrueux, génial, féerique » (MTR 205), ce Nestor ressemble à Tiffauges par son caractère « intemporel », il échappe comme lui « à la mesure du temps » (MTR 205). Comme Tiffauges, Nestor présente lui aussi deux faces: sa face manifeste qui se résume à « la trilogie ingestion - digestion - défécation » (MTR 207) et « sa face cachée » qui est décrite comme suit:

Sa face cachée [...] c'était les signes ${ }^{*}$, le déchiffrement des signes ${ }^{*}$. C'était là la grande affaire de sa vie [...]. Les signes, le déchiffrement des signes... De quels signes s'agissait-il? Que révélait leur déchiffrement? Si je pouvais répondre à cette question, toute ma vie serait changée, et non seulement ma vie mais - j'ose l'écrire assuré que personne ne lira jamais ces lignes ${ }^{9}$ - le cours même de l'histoire. Sans doute Nestor n'avait-il fait que quelques pas dans ce sens, mais ma seule ambition est précisément de mettre mes pieds dans sa trace $[\ldots] » .(\mathrm{MTR} 207)$

Quelques pages plus loin seulement, « la grande affaire de la vie [de Nestor] » est devenue la sienne. Au sujet de l'interprétation d'un tableau d'un Christ giflé, Tiffauges écrit:

\footnotetext{
${ }^{8}$ Le texte du roman ne nous donne aucun exemple de cette « écriture adroite » de Tiffauges.

9 Cette remarque laisse supposer qu'en écrivant son journal Tiffauges s'expose et s'exhibe à lui-même et non pas à autrui. Là encore Tiffauges ressemble à Nestor qui « prononc[e] [des] mots qui ne s'adress[ent] selon son habitude à personne » (MTR 64). Paroles en l'air ou plutôt paroles de méconnu clamées dans le désert et incomprises par le commun des mortels?
} 
J'étais encore si étranger à la lecture des signes - la grande affaire de ma vie - que je ne songeai pas au rapprochement qui s'imposait. Je sais aujourd'hui qu'un visage humain, aussi vil soit-il, souffleté, devient aussitôt la face de Jésus. (MTR 212)

Si la nature et l'interprétation des signes posent un problème à Tiffauges, il en est de même pour le lecteur. À plusieurs reprises dans le roman, Tiffauges, et avec lui le lecteur, se heurte à cette difficulté de compréhension des signes. Dès la deuxième page de son journal Tiffauges écrit qu'il le relit lui-même, et l'autoréflexion exprimée dans cette relecture, sa relecture, lui sert pour orienter et diriger l'interprétation de son journal par le lecteur supposé et par là pour s'emparer de l'interprétation par le lecteur. Pour Mariska Koopman-Thurlings « cette particularité du narrateur est une constante dans les romans de Tournier. Sa tactique consiste, non pas à présenter les événements pour que le lecteur puisse tirer ses conclusions, mais à le devancer en présentant des explications avant même que celui-ci ait pu arriver à une conclusion » (1995, pp. 161 et 191-192). ${ }^{10}$ Margaret Sankey constate à juste titre que Tiffauges est à la fois auteur, lecteur et interprète de ses Écrits sinistres (1991, pp. 325-340). Eeva Lehtovuori tire la même conclusion: « Le travail interprétatif du lecteur de Tournier se trouve immergé dans une auto-interprétation du discours lui-même, ce qui transforme, à son tour, le travail du critique en métainterprétation » (1995, pp. 4-5). L'analyse de David Gascoigne va dans le même sens: « In his 'Écrits sinistres', Abel Tiffauges cultivates and projects a certain image of himself, evolution and destiny as he sees it, and his narrative will develop a rhetoric of persuasion designed to recruite the reader into some degree of complicity with this view » (1996, p. 9). Ce procédé n'est donc pas gratuit: le caractère d'ogre de Tiffauges se répète et se confirme encore dans cette mainmise exercée par lui sur l'interprétation des Écrits sinistres par le lecteur. Avec le passage à la première personne du pluriel dans le fragment suivant Tiffauges semble en effet vouloir s'adresser à nous en tant que lecteurs de son journal et nous prendre ainsi à témoin, et par là nous entrâ̂ner et nous impliquer dans son existence:

Pour percer le mur de notre cécité et de notre surdité, il faut que les signes nous frappent à coups redoublés. Pour comprendre que tout est symbole et parabole de par le monde, il ne nous manque qu'une capacité d'attention infinie. (MTR $283)^{11}$

Ce passage confirme également la double forme auditive et visuelle des signes. ${ }^{12}$ Mais au risque d'une simplification trop poussée, nous sommes d'avis qu'une lecture approfondie du Roi des Aulnes permet également de distinguer globalement

\footnotetext{
10 Worton (1986) avait déjà signalé cette même propension de Tournier à gérer et diriger l'interprétation de ces romans par le lecteur.

11 Par l'expression de l'absence d' « une capacité d'attention infinie » ce fragment recoupe la phrase de Flaubert mise en exergue du roman: «Pour qu'une chose soit intéressante, il suffit de la regarder longtemps* ${ }^{*}(\mathrm{MTR} 191)$.

12 Cette dichotomie complémentaire correspond à l'idée fixe exprimée à plusieurs reprises par Tournier, qui aimerait passer inaperçu, ne pas être vu pour pouvoir tout voir et entendre.
} 
pour le fond deux catégories de signes qui s'imposent à Tiffauges. La première comprend ceux qui sont en rapport avec son (soi-disant) caractère exceptionnel et son (prétendu) « pouvoir surnaturel », signes qui sont plutôt d'ordre événementiel et dont l'interprétation par Tiffauges montre sa mégalomanie. ${ }^{13}$ La deuxième catégorie regroupe ceux qui relèvent de son écriture (sinistre) et des mots, signes d'ordre linguistique et littéraire. ${ }^{14}$ Distinction qui ne devra pas pour autant négliger les interférences et les interactions entre ces deux catégories. Les conclusions que nous pourrons tirer de l'analyse de ces deux types de signes nous conduiront dans un second temps à l'étude de l'onomastique du roman, les noms choisis par l'auteur et même ceux appartenant à l'Histoire ${ }^{15}$ étant autant de signes porteurs de messages à ne pas méconnaître.

\section{Les signes du caractère exceptionnel et du « pouvoir surnaturel » de Tiffauges}

Le (prétendu) caractère surnaturel de Tiffauges apparaît pour la première fois dans la scène de l'incendie du collège Saint-Christophe, qui coûte la vie à Nestor. Ayant fait une fugue auparavant, Tiffauges passe une nuit cauchemardesque craignant être puni à son retour au collège. Pour y échapper, l'image prémonitoire du collège en feu s'impose à lui, illusion qui devient réalité le lendemain à son arrivée. C'est alors que Tiffauges s'étonne et même s'énerve que l'on ne reconnaisse pas son «pouvoir naturel »:

Personne ne fit attention à moi, et je devais faire ainsi ce jour-là la première expérience de l'incroyable cécité des autres au signe fatidique qui me distingue entre tous. Il était donc possible d'ignorer la relation évidente, éclatante qui unissait cet incendie et mon destin personnel! Ces hommes stupides qui s'apprêtaient à m'écraser pour une peccadille - dont j'étais de surcroît innocent - ne reconnaîtraient jamais, quand même je leur hurlerais la vérité en pleine face, la part que j'avais dans le châtiment qui venait de frapper SaintChristophe! (MTR 245)

Cette méconnaissance accompagne Tiffauges pendant toute sa vie en France et y détermine son comportement social, à commencer par sa jeunesse à la maison et au collège Saint-Christophe. Une fois en Allemagne il se sent libéré de cette incompréhension. La scène en question est également significative pour la façon dont Tiffauges lie et plie les événements, historiques ou non, à son destin personnel: il considère que l'incendie est une nécessité pour le sauvegarder de la punition. Dès la première page des Écrits sinistres cette conviction est annoncée: « Je crois, oui, à ma nature féerique, je veux dire à cette connivence secrète qui mêle en profondeur mon aventure personnelle au cours des choses, et lui permet de l'incliner dans son sens »

\footnotetext{
13 Voir aussi Jean-Bernard Vray (1997, pp. 76-80, 88).

14 Pour Liesbeth Korthals Altes (1992, pp. 45-49 et 58) le déchiffrement des signes et l'écriture ou la production des signes sont deux faces d'une même « manipulation des signes », ces deux faces étant aussi complémentaires.

15 Nous écrivons Histoire avec majuscule pour la distinguer de l'histoire, du récit fictif du roman.
} 
(MTR 291). De la même manière mais dans un autre ordre de grandeur, l'éclatement de la Seconde Guerre mondiale aura lieu, dans l'optique de Tiffauges, pour le sauver de la prison. ${ }^{16}$ Vladimir Tumanov résume cette vision des événements par Tiffauges comme suit: « Tiffauges is convinced that the world revolves around him and therefore views various events as signs that concern him alone » et « [...] however marginalized he may feel in society, Tiffauges, as ogre, affects the course of the world's events [...], and it is [the] mythologization of his own marginality that gives Tiffauges the idea that he is at the center of everything » (1999). La marginalité de Tiffauges se trouve ainsi compensée par son (prétendu) génie visionnaire.

Si Nestor est mort par le feu, il renaît dans la personne de Tiffauges qui ainsi « reprend la grande affaire de sa vie » (MTR 208), renaissance qui fait penser au mythe du phénix resurgissant de ses cendres. Cette scène de la mort de Nestor asphyxié près de la chaudière de l'internat semble annoncer les pages sur cet autre espace clos racontées par Éphraïm à la fin du roman, celui du camp d'extermination où les Juifs sont gazés. Le remplacement de Nestor par Tiffauges se situe au niveau physique et mental: rachitique pendant son enfance, Tiffauges devient aussi gros et vorace que Nestor avant de mourir et le développement intellectuel de Nestor est transmis à Tiffauges qui était nul pendant sa scolarité (voir MTR 248). Mais l'élément le plus évident pour cette relève est bien sûr l'écriture de la main gauche de Nestor (MTR 216) reprise par Tiffauges dans ses Écrits sinistres.

Tiffauges se prétend « environné de signes et d'éclairs » (MTR 246) et c'est dans le contexte de « tous les signes qui pouvaient apparaître » (MTR 248) que Tiffauges adulte se compare implicitement à Cagliostro et Raspoutine, deux figures historiques énigmatiques et mythiques qui sont entrées dans la légende par leurs prétendus pouvoirs surnaturels. Tiffauges se dit porteur d'une « force ténébreuse » mais avoue encore son impuissance à la cerner: « Moi-même j'ai peine à concevoir ce secret de mon destin » (MTR 259).

Dans la deuxième partie du roman Tiffauges passe la main à un narrateur omniprésent. Avec une apparente objectivité ${ }^{17}$ celui-ci confirme textuellement les difficultés de Tiffauges à traiter les signes, bien qu'il s'agisse de signes d'un autre niveau:

En janvier 1940, son incapacité à maîtriser des signes conventionnels, abstraits, futiles, sans charge fatale, fut sanctionnée par son échec à l'examen de caporal, et ce fut comme soldat de deuxième classe qu'il fut envoyé à Erstein [...].

(MTR 310)

Contraste bien dur: lui qui se voit à l'origine des grands événements dans le monde reste au plus bas dans la hiérarchie militaire française, précisément à cause de son

\footnotetext{
16 Dans une interview Tournier déclare: «J'étais content que la guerre éclate. C'était une réaction de sale gosse qui voyait dans la crise générale un moyen d'échapper à sa crise personnelle. Je ressuscite cet épisode dans Le Roi des Aulnes, quand Abel Tiffauges parvient à échapper aux assises grâce à la déclaration de guerre! » (2011, p. 32).

17 Que cette objectivité soit juste ou pas, le lecteur est à nouveau manipulé, car le narrateur authentifie de manière claire et nette le statut et le contenu des Écrits sinistres.
} 
impuissance à interpréter les signes... Mais il convient bien de se rendre compte que les qualificatifs « conventionnels, abstraits, futiles, sans charge fatale » désignent des signes de qualité inférieure, étrangers aux signes d'ordre supérieur auxquels semble être destiné Tiffauges. ${ }^{18}$ La supériorité de ces signes se définit par leur union avec la chair:

Tiffauges [était] [...] ancr[é] peu à peu dans l'idée que la guerre n'était qu'un affrontement de chiffres et de signes, une pure mêlée audio-visuelle sans autre risque que des obscurités ou des erreurs d'interprétation. Personne n'était mieux préparé apparemment que lui à ces problèmes de réception, de déchiffrement et d'émission. Pourtant ils lui demeuraient étrangers, car, dépourvus de l'élément vivant, chaleureux et sanguin qui était pour lui comme la signature de l'être, ils flottaient dans une sphère abstraite, contemplative et gratuite. Il attendait avec confiance et patience cette union du signe et de la chair qui était pour lui la fin dernière des choses, et singulièrement de cette guerre. Elle devait lui être offerte quelques semaines plus tard, sous une forme certes dérisoire, mais non moins annonciatrice d'accomplissements ultérieurs (MTR 312).

Cette union du signe et de la chair a été signalée aussi dans le journal de Tiffauges, qui écrit «les signes ont besoin de la chair pour se manifester* » (MTR 280). Elle ne saurait surprendre dans ce récit d'ogre où le rôle de la chair est évident et pertinent. La « forme dérisoire » dont il est question dans ce fragment est celle des pigeons: " des messagers fidèles, des porte-signes vivants et palpants » (MTR 325) appelés encore « petits porte-signes » quelques pages plus loin (MTR 327). Les pigeons dont s'occupe Tiffauges symbolisent cette union du signe et de la chair: ils sont signes en chair et en os. Notons enfin que les signes colombins sont écrits. Dans son « Prière d'insérer de l'Édition originale » Tournier insiste sur ce rapport indissociable entre le signe et la chair:

Deux passions éclairent et réchauffent [1]a solitude [de Tiffauges]: la détection des symboles dont il devine la présence autour de lui, et le goût de la chair fraîche. ${ }^{19}$

Ce qui vaut pour les signes comme indications et preuves du caractère surnaturel de Tiffauges, s'applique également aux notions tiffaugéennes de symbole et d'allégorie. Ainsi il donne à ses difficultés respiratoires, ennuis physiques appelés par lui «oppression angélique*, ou plus brièvement angélique* », une « signification fondamentale », issue « d'une relation de symbole à chose symbolisée »:

Grâce à [cette signification fondamentale], mes poumons sont passés de la nuit glandulaire à la pénombre viscérale, voire, dans les cas extrêmes, à la grande lumière de la conscience. Ces cas extrêmes, ce sont la grande angoisse dyspnéique qui me fait lutter sur le sol contre une étreinte invisible et meurtrière, mais aussi

18 Voir aussi à ce sujet David Platten (1991, pp. 292-293).

19 «Prière d'insérer de l'Édition originale », dans Tournier (2017, MTR 542). 
la profonde et bienheureuse aspiration par laquelle le ciel tout entier plein de vols d'hirondelles et d'accords de harpe ${ }^{20}$ plonge directement dans mes poumons sa racine bifurquée. (MTR 253)

Une fois arrivé en Allemagne, Tiffauges est toujours aussi voire encore plus attentif aux signes (MTR 356): cette terre lui inspire la sublimation de cette conscience « fondamentale ». Il se dit que ce pays donne une tournure positive à son pouvoir surnaturel, là où il était resté dans les ténèbres avant:

[P]our Tiffauges dont le ciel clouté d'allégories et d'hiéroglyphes retentissait sans cesse de voix indistinctes et de cris énigmatiques, l'Allemagne se dévoilait comme une terre promise, comme le pays des essences pures ${ }^{*}$. (MTR 350) ${ }^{21}$

Rejeté par la France ${ }^{22}$ où se trouvaient concentrés tous ses malheurs, tout son malaise et toute sa méconnaissance, Tiffauges, en utilisant le terme de « promise », confère ainsi une dimension biblique à cette terre révélatrice qui lui a été « donné[e] par le destin » (MTR 350) et qu'il se donne pour tâche d'interpréter et d' " élev[er] à une puissance supérieure $»$ :

Et voici qu'il avait la révélation que la Prusse-Orientale tout entière était une constellation d'allégories, et qu'il lui appartenait de se glisser en chacune d'elles [...]. Car il n'avait pas seulement vocation de déchiffrer les essences, mais aussi de les exalter, de porter toutes leurs vertus à incandescence. Il allait livrer cette terre à une interprétation tiffaugéenne, et en même temps, il l'élèverait à une puissance supérieure, encore jamais atteinte. (MTR 351)

Tiffauges va même jusqu'à creuser farouchement et fouiller littéralement la terre de ce pays prometteur, dont « il attendait quelque chose* », « un signe, un présage, il ne savait au juste », étant toujours persuadé que ce message délivré par cette terre était « à lui seul destiné » (MTR 338). ${ }^{23}$ Force est cependant de constater que la place exceptionnelle dans ce monde en pleine ébullition que croit occuper Tiffauges et le caractère surnaturel qu'il désire se conférer ne lui permettent d'interpréter que difficilement et imparfaitement tous ces signes. La myopie, ${ }^{24}$ la vue courte dont souffre Tiffauges sur le plan

\footnotetext{
$\overline{{ }^{20}}$ On relèvera encore les aspects visuels par le « vol d'hirondelles » et auditif par les « accords de harpe ».

21 Voir aussi 1'article d'Anthony Purdy (1993, pp. 21-33).

${ }^{22}$ Le narrateur se livre à toute une comparaison entre la France et l'Allemagne, en précisant ce que Tiffauges «était venu chercher si loin vers le nord-est: sous la lumière hyperboréenne froide et pénétrante tous les symboles brillaient d'un éclat inégalé* »(MTR 250).

${ }^{23}$ L'ogre qui se nourrit de viande rouge crue et qui creuse la terre à la recherche de signes est exemplaire pour la concrétisation du concept nazi de Blut und Boden. Voir aussi MTR 437 et l'étude de Krell (1994, pp. 66-70).

${ }^{24}$ Il n'est pas précisé dans le roman si Tiffauges souffre aussi d'un strabisme. Toujours est-il que John Malkovich, qui joue le rôle de Tiffauges dans le film tiré du roman, présente bel et bien une coquetterie dans l'œil. Il est tentant de mettre la myopie et l'éventuel strabisme de Tiffauges en rapport avec le sens de louche synonyme de sinistre retenu comme qualificatif pour son journal «Écrits sinistres* ${ }^{*}$. Notons aussi que la myopie de Tiffauges est compensée par le développement des autres sens: l'ouie, l'odorat, le goût et le toucher.
} 
physique, se double en effet d'un manque de perspicacité au niveau mental. Malgré l'intimité et la confidentialité ${ }^{25}$ des échanges avec le comte de Kaltenborn, l'explication des symboles donnée par celui-ci ne semble pas éclairer Tiffauges dans sa recherche de l'interprétation des signes. Pourtant le «Kommandeur* ${ }^{*}$ a parfaitement compris la nature et les intérêts de Tiffauges, qu'il appelle « un lecteur de signes » et « un homme marqué par le destin ». Si les propos tenus par Herbert von Kaltenborn recoupent même parfois textuellement les écrits de Tiffauges (« [t] out est dans les symboles ») (MTR 460), ils font preuve aussi d'une volonté et d'une disponibilité de faire avancer et d'instruire Tiffauges:

Jusqu'ici vous avez découvert des signes sur les choses, comme les lettres et les chiffres qu'on lit sur une borne. Ce n'est que la forme faible de l'existence symbolique. Mais n'allez pas croire que les signes soient toujours d'inoffensives et faibles abstractions. Les signes sont forts, Tiffauges, ce sont eux qui vous ont amené ici. Les signes sont irritables. Le symbole bafoué devient diabole. ${ }^{26}$ (MTR 460)

En évoquant l'Apocalypse de Saint Jean, le Kommandeur apprend à Tiffauges que la fin du monde approche:

Et tout cela (=les scènes décrites dans l'Apocalypse) est symbole, tout cela est chiffre, ${ }^{27}$ indiscutablement. Mais ne cherchez pas à comprendre, c'est-à-dire à trouver pour chaque signe la chose à laquelle il renvoie. Car ces symboles sont diaboles: ils ne symbolisent plus rien. Et de leur saturation naît la fin du monde. (MTR 461)

«Vous aimez la Prusse, monsieur Tiffauges, parce que sous la lumière hyperboréenne, dites-vous, les signes brillent d'un éclat incomparable. Mais vous ne voyez pas encore où mène cette prolifération redoutable de symboles. Dans le ciel saturé de figures se prépare un orage qui aura la violence d'une apocalypse, et qui nous engloutira tous ! ». (MTR 463)

Jonathan Krell a analysé ce concept de « diabole ». Pour lui, « [u]n diabole est l'inversion maligne d'un symbole. Le propre d'un symbole est sa polysémie: il possède à un premier niveau un sens manifeste, et à un second niveau des sens latents, cachés. [...] Le diabole est le symbole vidé de son sens caché, un symbolisant sans symbolisé $»{ }^{28}$ Tiffauges donne l'impression de ne rien comprendre de ce discours du comte de Kaltenborn, il n'en tire aucune leçon, dans l'immédiat du moins. Pour qu'il le fasse enfin, il lui faudra la rencontre avec un enfant, enfant victime précisément de cette Allemagne nazie maligne et destructive. C'est en effet seulement vers la fin du roman que Tiffauges y voit plus clair en écoutant « de toutes ses oreilles » (MTR 505) Ephraïm, qui lui ouvre les yeux de ses lumières. Ainsi ce ne sont pas

\footnotetext{
${ }^{25}$ Cependant Tiffauges, qui « ne lui (=le comte de Kaltenborn) avait rien révélé de sa race ogresse, ni de la complicité qui l'unissait au destin », se déclare avide « d'en apprendre davantage » (MTR 459).

26 Il s'agit ici d'un néologisme qui combine « symbole » et « diable ».

27 Notons l'étymologie du mot chiffre qui remonte à l'arabe sifr dont le sens est vide ou zéro. Voir à ce sujet Jiménez (2016, pp. 327-337).

28 Krell (1994, pp. 188-189).
} 
les adultes, mais trois enfants qui déterminent la vie et la mort de Tiffauges: après Nestor au début du roman, c'est Martine, la fillette qui prétend avoir été violée par Tiffauges. Vu la sincérité manifeste de Nestor et d'Éphraïm ainsi que la franchise générale attribuée aux enfants dans l'œuvre de Tournier, on serait tenté de reconnaître la véracité de l'accusation de Martine et donc de conclure à la culpabilité de Tiffauges dans cette affaire du viol où plane toujours le doute. En tout cas, la mystification et par là la manipulation du lecteur par Tiffauges narrateur bat son plein dans cet épisode. C'est en effet Ephraïm, « [L'] enfant Porte-Étoile » (MTR 504), qui lui révèle, seulement à la fin du roman, « dans un yiddish mêlé de mots hébreux, lituaniens et polonais » les horreurs des camps de concentration, « monde souterrain sans rapports - autres qu' accidentels - avec le monde superficiel des vivants « situé » sous cette Allemagne, tout entière exaltée et polarisée par la guerre » (MTR 505). Il est à noter que l'attrait du nazisme reposait pour une très grande partie sur l'emploi voire l'exploitation des symboles que ses dirigeants puisaient de préférence dans l'histoire, la tradition, la légende, le rite et la cérémonie. Avec sa théorie sur « l'antiquité vertigineuse de [s] es origines » (MTR 191) et ses pratiques et habitudes rituelles particulières, du brame par exemple, Tiffauges s'inscrit directement dans cette même lignée.

Le monde de Tiffauges s'écroule « [en voyant] s'édifier un univers qui reflétait le sien avec une fidélité effrayante et qui en inversait tous les signes » (MTR 505). C'est ainsi que le thème des signes rejoint celui de l'inversion, qui est tout aussi récurrent dans le roman. Cette description de la double face de l'Allemagne s'apparente à celle utilisée à plusieurs reprises par Tiffauges pour définir la photographie. L'opposition entre le noir et le blanc, entre le négatif et le positif, l'un étant le reflet inversé de l'autre, parcourt tout le roman sinon toute l'œuvre de Tournier. ${ }^{29}$ L'univers de Tiffauges se définit aussi par le négatif, par ce qu'il n'est pas, univers glacial, noir et boueux où Tiffauges s'enfonce au sens figuré et littéralement, portant Éphraïm sur ses épaules. C'est l'échec de « [l']inversion bénigne [qui] consiste à retourner le sens des valeurs que l'inversion maligne a précédemment retourné » (MTR 257). La myopie de Tiffauges qui lui a interdit pendant toute sa vie de comprendre les signes et les symboles se résout dans le récit des expériences d'Éphraïm:

Abreuvé d'horreur, Tiffauges voyait ainsi s'édifier impitoyablement, à travers les longues confessions d'Éphraïm, une Cité infernale qui répondait pierre par pierre à la Cité phorique dont il avait rêvé à Kaltenborn. Le Canada, le tissage des cheveux, les appels, les chiens dobermans, les recherches sur la gémellité et les densités atmosphériques, et surtout, surtout les fausses salles de douche, toutes ses inventions, toutes ses découvertes se reflétaient dans l'horrible miroir, inversées et portées à une incandescence d'enfer. Il lui restait encore à apprendre que les deux peuples sur lesquels s'acharnaient les S.S., et dont ils poursuivaient l'extinction, étaient les peuples juif et gitan. Ainsi, il retrouvait ici poussée à son paroxysme la haine millénaire des races sédentaires contre les races nomades. Juifs et gitans, peuples errants, fils d'Abel, ces frères dont il

${ }^{29}$ Voir à ce sujet par exemple son Gilles and Jeanne. 
se sentait solidaire par le cœur et par l'âme, tombaient en masse à Auschwitz sous les coups d'un Caïn botté, casqué et scientifiquement organisé. La déduction tiffaugéenne des camps de la mort était achevée. (MTR 509)

Les signes « événementiels » et leur interprétation aboutissent ainsi à la désillusion, la désorientation ${ }^{30}$ totale et à l'apocalypse. Nous verrons que les signes écrits vont dans le même sens, tout en ouvrant la voie à une interprétation qui double sinon dépasse celle qui s'impose à première vue.

\section{L'écriture, autant de signes à interpréter}

Il est difficile de déterminer la nature des hiéroglyphes qui se présentent à Tiffauges à trois reprises, deux fois dans son journal (MTR 193 et 274) et une fois dans la partie III du roman (MTR 350). C'est le deuxième fragment qui paraît le plus intéressant quant aux réflexions livrées par Tiffauges. Muni de son appareil photo, il observe les enfants qui jouent dans la cour de récréation du collège Sainte-Croix, à travers la grille. Il constate et répète que

[...] tout est signe ici, comme ailleurs, davantage qu'ailleurs. Mais signe de quoi ? C'est mon éternelle question dans ce monde semé d'hiéroglyphes dont je n'ai pas la clé. (MTR 274)

Ces « hiéroglyphes » sont consignés par Tiffauges qui les prend en photo pour les étudier et analyser tranquillement chez lui plus tard, et ce toujours dans le même objectif d'y « découvr[ir] quelque chose » (MTR 274). Toute cette scène est remplie de comparaisons. Ainsi les grilles de la cour sont comparées à celles d'une cage et la « rafale de photos » des enfants à laquelle se livre Tiffauges est mise en rapport avec des balles tirées sur des bêtes par un chasseur. ${ }^{31}$ Le caractère d'ogre est évoqué explicitement ici sous la plume de Tiffauges:

Mettre des enfants en cage... Mon âme ogresse y trouverait son compte. Mais il y a autre chose qui va plus loin qu'un simple jeu de mots. Toute grille est grille de déchiffrement, il n'est que de savoir l'appliquer. (MTR 274)

L'immobilisation des « hiéroglyphes » au moyen de la photographie ${ }^{32}$ ne se compare-t-elle pas à la consignation des événements par l'écriture? Appareil photo ou plume, tous deux ne servent-ils pas à essayer de surmonter la myopie et d'y voir plus clair? Pour Tiffauges, mais aussi, indirectement, pour « son » lecteur ! Et tout comme la pellicule sensible est indispensable à la photographie, le papier ou la page blanche l'est à l'écriture. C'est au sujet des jeux dans cette autre cour de récréation, celle du collège Saint-Christophe, que Tiffauges cite Nestor:

\footnotetext{
30 Cette désorientation est à prendre au pied de la lettre: elle explique pourquoi Tiffauges se laisse guider « sans discuter» (MTR 521) par Éphraïm à la fin du roman.

31 Le thème de la chasse est bien sûr inséparable de celui de l'ogre. Nous y reviendrons.

${ }^{32}$ La photographie, on l'a vu, s'inscrit aussi dans la thématique de l'inversion, l'image positive étant consignée sous sa forme négative.
} 
«Une cour de récréation, dit-il (=Nestor), c'est un espace clos qui laisse assez de jeu pour autoriser les jeux. Ce jeu est la page blanche où les jeux viennent s'inscrire comme autant de signes qui restent à déchiffrer. Mais la densité de l'atmosphère est inversement proportionnelle à l'espace qui l'enferme. Il faudrait voir ce qui se passerait si les murs se rapprochaient. Alors l'écriture se resserrerait. En serait-elle plus lisible? À la limite on assisterait à des phénomènes de condensation. Quelle condensation? Peut-être l'aquarium, ${ }^{33}$ et mieux encore les dortoirs, pourraient-ils fournir une réponse ». (MTR 229)

Ce passage est à rapprocher d'un autre fragment du journal consacré à la vie du dortoir, encore un espace clos du collège Saint-Christophe. Tiffauges cite alors Nestor, qui, encore, «se parl[e] volontiers à lui-même » : «- Ici, disait-il, la concentration est extrême. Le jeu est réduit autant qu'il se peut. Le mouvement s'est figé en des attitudes qui varient certes, mais avec une lenteur infinie. N'importe, ce sont là autant de figures qu'il faudrait lire. Il doit y avoir un signe absolu alpha-oméga. Mais où le trouver ? » (MTR 239). «Densité », « resserrer », « condensation », « concentration », « réduire », tous ces termes convergent, dans le sens qui pourrait bien être celui de l'auteur à la recherche de la clef universelle et existentielle pour exprimer sa pensée en la couchant sur papier. C'est aussi en évoquant le legs de Nestor que Tiffauges témoigne de ce rapport indissociable entre papier et écriture en « regard[ant] [s]a main courir sur le papier, [s]a main gauche tracer les lettres successives de cet écrit « sinistre ${ }^{34}$ (MTR 216). À son arrivée à la napola ${ }^{35}$ de Kaltenborn, Tiffauges est confronté à une variante de ce support du papier à l'écriture, à savoir les « murs [qui] parlaient et criaient en devises et en aphorismes, chantaient en drapeaux et oriflammes, comme si ce fût à eux seuls que fût dévolue la faculté de penser » (MTR 403).

Dans ce même registre s'inscrit le curieux fragment où il est question d'un homme qui se met en tête d'incendier ${ }^{36}$ des lieux publics afin de détruire tous les dossiers personnels et officiels. Dans cette anecdote appelée « apologue » par Tiffauges (MTR 223), fable qui se présente cependant comme un conte ${ }^{37}$ car il commence par «Il était une fois [...] », les hommes privés de leurs papiers «se métamorphos[ent] en bêtes »(MTR 223). Cette privation de papiers d'identité qui déshumanise peut se lire comme une métaphore: l'homme a besoin de papier afin d'exister, comme l'écrivain a besoin de papier pour écrire et le lecteur pour lire « parce que l'âme humaine est en papier* ${ }^{*}(\mathrm{MTR} 223) .{ }^{38}$

\footnotetext{
33 L'aquarium est le jardin clos par les galeries du cloître Saint-Christophe qui sert de cour de récréation. Ce lieu est décrit aussi par Tiffauges comme « prison verte » (MTR 209).

34 Entre guillemets dans le texte.

35 Napola = nationalpolitische Erziehungsanstalt.

36 Des incendies, toujours des incendies ! Voir au sujet du rôle du feu dans l'œuvre de Tournier: Krell (1994, pp. 16-22 et 118-126).

37 C'est le terme utilisé par Tournier pour appeler cette histoire. Voir Bouloumié (1989, p. 151).

38 Notons aussi qu'à l'inverse le passage de la tradition orale, domaine privilégié du conte, à la tradition écrite correspond à l'humanisation dans le sens où, avec l'écrit, le féerique et le mythique perdent du terrain au niveau bestial au profit du réel et du vraisemblable.
} 
À la vérité, la terminologie utilisée dans le roman pour comprendre et interpréter les signes, écrits ou autres, est souvent basée sur l'écriture, mais aussi sur son complément, la lecture. Ainsi Tiffauges, dans lequel le comte de Kaltenborn dit avoir reconnu « un lecteur de signes » $(\mathrm{MTR} 460)$, écrit dans son journal qu'il a repris dans la partie $\mathrm{V}$ du roman:

[...] je me trouve ici (=en Allemagne) constamment confronté à une réalité signifiante* presque toujours claire et distincte, ou alors quand elle devient difficile à lire, c'est qu'elle s'approfondit et gagne en richesse ce qu'elle perd en évidence. (MTR 421).

Nous sommes d'avis que cette réflexion s'applique à tout le roman, qui lui aussi est « clair et distinct » ou encore « éviden[t] » par endroits, mais qui présente également des éléments moins limpides et moins explicites qui n'en sont que plus profonds et plus riches. C'est en répondant à cet appel de double voire multiple lecture que nous analyserons l'onomastique dans le roman, les noms propres présentant des signes évidents et moins évidents à interpréter et à intégrer dans la compréhension globale du roman.

\section{L'onomastique}

Dans Je m'avance masqué Tournier exprime à plusieurs reprises l'importance des noms propres et de leur signification pour lui. D'abord au sujet des pseudonymes et des noms juifs:

Oui, j'ai pensé à un pseudonyme en inversant mon nom et en supprimant le $u$. Cela donnait Edward Reinrot, un nom juif allemand qui signifie « rouge pur ${ }^{39}$

[Les juifs] ont pris les meilleurs noms: Gut, Reich, Blum, Rose, Freud, Stein, Gold. Les juifs ont des noms extraordinaires. Gutmann: l'homme du bien. Pas mal ! ${ }^{40}$

Il montre aussi son émerveillement devant la beauté des noms et sa conscience de l'influence qui en dépend:

Il y a des gens qui n'auraient pu faire la carrière qu'ils ont faite s'ils avaient conservé leur nom. Pensez à Fred Astaire. Jamais il n'aurait pu mener sa carrière [...] s'il était resté Frederick Austerlitz. [...] Et le Caravage, un nom merveilleux! Que ne donnerais-je pour le porter! [...] C'est autre chose que Tournier $!^{41}$

\footnotetext{
39 Tournier (2011, p. 109). Voir aussi nos remarques sur le nom de Reinroth (infra).

40 Ibid., p. 81.

41 Ibid., p. 109. Voir aussi nos remarques sur les noms allemands (infra). Dans Pourquoi je n'ai pas changé de nom, Michel Tournier s'explique sur le phénomène des pseudonymes. Relevons notamment celui du mystique Angelus Choiselus (Choisel étant le nom du village où il habite). Signalons aussi que le nom de Tournier et, par paronomase, le verbe « tourner » s'inscrivent dans le même champ sémantique que celui de l'inversion, au niveau thématique et anagrammatical.
} 
Le prénom de Goethe et de Mozart, Wolfgang, m'a toujours intrigué. J'ai découvert qu'il signifie « le pas du loup ». Or nous allions aux Concerts Pasdeloup, du nom de son chef, qui était la traduction française du prénom de Mozart ! ${ }^{42}$

Le nombre de noms propres dans Le Roi des Aulnes est élevé et se compose de prénoms, patronymes, surnoms et noms géographiques. David Bevan ${ }^{43}$ associe le déchiffrement de certains mots et noms dans l'œuvre de Tournier au sens de l'humour de celui-ci: «Un clin d'œil comique n'est jamais très loin chez un auteur qui est naturellement débordant d'humour. Mais combien subtils et fugitifs parfois sont certains 'mots' qu'il faut essayer de saisir au vol ». Il convient de reconnaître que certains noms tourniériens sont bien éloquents: Abel Tiffauges, Alexandre Surin, Raphaël Bidoche, Logre, Tupik, ${ }^{44}$ Lucien Gagneron, ${ }^{45}$ Thomas Koussek, Véronique, ${ }^{46}$ Étienne Milan, ${ }^{47}$ Frédéric Durâteau, Tristan Vox, ${ }^{48}$ Henri Durieu, ${ }^{49}$ etc., montrant ainsi la nette prédilection de Tournier pour des noms forgés et porteurs de sens. Jean-Luc Mercié indique « [qu']il semble que Tournier ait emprunté deux procédés à Flaubert: le jeu onomastique et la mise en scène d'expressions lexicalisées ». Pour Mercié « [1] e nom propre, sémantiquement surchargé, est à double, voire à triple entente »(1991, pp. 252-254). Thierry Miguet présente quelques exemples assez surprenants de l'onomastique tourniérienne qui porte sur le A et le O inspirés par l'Alpha et l'Oméga (1991, pp. 179-182). Dans cette même optique Jean-Bernard Vray consacre un chapitre fort intéressant à la multiple présence de la combinaison des lettres O et R dans l'œuvre de Tournier (1997, pp. 385-389).

Comme plusieurs autres critiques, Liesbeth Korthals Altes a signalé cette propension de Tournier pour les jeux de mots, dont fait partie bien entendu le jeu onomastique (1992, p. 155). Pour notre part l'exemple le plus caractéristique et le plus évident de ce penchant de Tournier dans Le Roi des Aulnes est le jeu de mots sur les forts* et les phores* des Halles. Ce jeu de mots est encore doublé par un jeu sur le jugement de valeur orthographique: « Le fort est la forme vulgaire du phore », comme si l'écriture avec « ph » était donc la forme plus noble (MTR 264). L'auteur épuise toutes les expressions possibles se rapportant à la phorie, thème principal du

\footnotetext{
42 Ibid., p. 193. Cet orchestre symphonique a été fondé en 1861 par Jules Pasdeloup. Notons aussi que Tournier se montre extrêmement sensible à ce type de coïncidences. Voir nos remarques sur Kaltenborn/ Kaltenbronn et Abel Tiffauges/Philippe Janssen (infra), comme celles sur les dates d'événements ou d'anniversaires qui coïncident (infra).

43 Bevan (1986, pp. 57-62 et 64-65).

44 Tupik $\approx$ tu piques, en référence à la barbe de son père rébarbatif. Mais Tournier savait-il que « pik » signifie « bite » en néerlandais quand il a choisi le nom de Tupik pour ce personnage qui procède à sa castration?

45 Gagneron $\approx$ Gag-Néron, Lucien exécutant une parodie d'empereur romain au cirque.

46 Veronique $\approx$ vera icon.

47 Double connotation: Milan, centre de mode avec ses mannequins (objets d'Étienne photographe), et mille ans, l'intemporalité du cliché.

48 Tristan Vox $\approx$ « spiqueur » à la radio.

49 Henri Durieu $\approx$ opposition « Dieu - rien », anagramme signalée par Klettke (1993, pp. 47-61).
} 
roman: euphorie, paraphorie, superphorie, astrophorie. Sans compter les innombrables apparitions de combinaisons avec le verbe porter. ${ }^{50}$ L'euphorie ressentie et exprimée par Tiffauges quand il porte un enfant est un jeu de mots qui sous-tend tout le roman: cette expression est à prendre littéralement, avec tous les enfants portés par Tiffauges dans le roman, à commencer par son aide garagiste Jeannot (MTR 261). ${ }^{51}$ Mais elle doit être comprise aussi dans le sens de la femme qui porte, en elle, un enfant pendant sa grossesse, le côté maternel de Tiffauges étant ainsi suggéré par l'ambiguiité du jeu de mots. ${ }^{52}$

Tournier affirme son amour du calembour: « [...] je crois en la profondeur voilée d'ironie du calembour. Sahara - Canada. Ces deux mots de six lettres dont trois $a$ placés aux mêmes points sont d'une saisissante analogie » $\left(1977\right.$, p. 52) ${ }^{53}$ Platten (1999b, p. X, préface) relève lui aussi le goût de Tournier pour le jeu de mots, leurs effets et utilité: 54

[...] the pun is eulogized because it brings to the fore two different meanings at the same time and not because its casts doubt on one or both of the meanings expressed or on the integrity of language as a vehicle of communication. The pun is thus seen as a figure which amplifies knowledge rather than fractures meaning.

En s'appuyant sur Bouloumié (1988, pp. 73-81), ${ }^{55}$ Korthals Altes (1992, p. 155) évoque le rapport entre le calembour et la fugue, dont la structure est sous-jacente à la composition du Roi des Aulnes, comme à celle des mythes. Dans ce contexte elle cite Claude Lévi-Strauss, qui compare musique et mythe pour constater que, comme la fugue notamment, les mythes se composent « [d]'images qui s'inversent de positives en négatives, se retournent de droite à gauche ou de haut en bas ; transformations qui rappellent le mécanisme du calembour [et dont la] seule limite [est] ce qu' [...] on pourrait appeler 1 [eur] 'capacité anagrammatique' » $\left(1971\right.$, p. 581) ${ }^{56}$ Tournier souligne lui-même son admiration sans limite de l'Art de la fugue de JeanSébastien Bach, qu'il déclare « [l']œuvre musicale la plus riche, la plus rigoureuse,

\footnotetext{
50 Tournier s'en explique dans Le Vent Paraclet, en jouant encore sur les mots: « Qui porte l'enfant l'emporte. Qui le sert humblement le serre criminellement» (MTR 1397). Voir aussi nos remarques infra sur Saint-Christophe et Porte-des-Ternes.

51 Datant de trois jours plus tard, la réapparition dans le roman du mot euphorie est relatée à cet événement (MTR 262).

52 Le cadre du présent article ne permet pas d'entrer dans le sujet du caractère maternel (ou androgyne) de Tiffauges. Contentons-nous d'évoquer la dernière partie du roman où Tiffauges recueille Éphraïm et s'en occupe comme une mère.

53 On a du mal à comprendre l'affirmation de Robert Sabatier, ami de Tournier, selon laquelle « [celuici] déteste les calembours et la forme d'humour joyeuse et blagueuse qui est la mienne » (Tournier 2011, p. 216).

54 Cette utilité n'est pas que littéraire: dans une lettre adressée à Gérard Depardieu Tournier tente de convaincre l'acteur à jouer le rôle principal de son roman en écrivant « [c]e film est le film le plus depardivin qui soit » (Bouloumié (2016, p. 187)).

55 Voir aussi Tournier (2011, p. 246).

56 Nous reviendrons in extenso sur le rôle de l'anagramme dans Le Roi des Aulnes.
} 


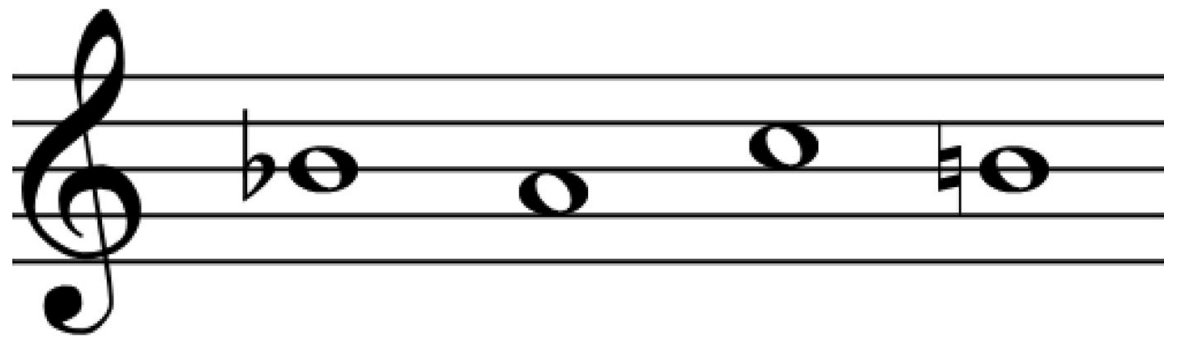

Fig. 1 Le motif BACH. Wikimedia Commons

la plus touchante qui fut jamais conçue de tête humaine et réalisée de main humaine, l'idéal insurpassable de toute création [...] » ${ }^{57}$ (2017, MTR 1399). Qui plus est, il rappelle le jeu anagrammatique auquel s'est livré Bach au niveau des lettres de son nom et de l'annotation de sa composition BWV 1080, réalisant ainsi par sa signature musicale une fusion totale entre patronyme et création (Fig. 1). En d'autres termes, anagrammatiquement le nom de Bach et le thème de sa musique sont indissociables, de manière intrinsèque bien entendu:

Dans la onzième fugue nous sommes avertis de l'approche du dénouement par l'apparition d'un thème dont les notes ( $s i$ bémol, la, do, si bécarre) correspondent selon la notation allemande aux quatre lettres du nom BACH. Le dénouement en effet, car l'homme vient d'être dévoré par son œuvre, et cet ultime sacrifice ne peut être dépassé. Le thème $\mathrm{BACH}$ est repris en contresujet dans la quinzième fugue, inachevée celle-là, dont le manuscrit porte ces mots écrits de la main de Carl-Philippe-Emanuel Bach Le compositeur a été trouvé mort sur cette fugue où le nom de BACH apparaît en contre-sujet*. (Le Vent Paraclet, MTR 1400)

L'émerveillement de Tournier devant la structuration de l'œuvre musicale de Bach et le mécanisme anagrammatique de celui-ci pour y confondre son patronyme en dit long sur sa conception de l'onomastique significative. Petit (1986, pp. 232-233) avance l'hypothèse de la structuration du Roi des Aulnes sur ce même principe de la fugue où se reconnaîtraient successivement les huit thèmes ${ }^{58}$ du roman, chacun commençant par les lettres formant le nom de TOURNIER:

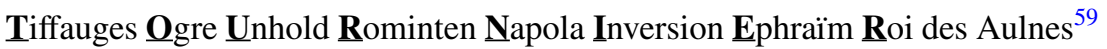

L'intérêt de Tournier pour (l'étymologie de) l'onomastique est établi et confirmé par la plupart des tourniérologues. Voici un rapide aperçu de leurs recherches et quelques conclusions fort intéressantes qu'ils ont pu en tirer.

\footnotetext{
57 Voir aussi Klettke (1993, pp. 47-66); Gasgoigne (1996, pp. 12-14 et 207) et Arlette Bouloumié dans Tournier (2011, p. 246).

58 Notons cependant qu'il n'est question que de six chapitres dans la structure formelle du roman et que leurs titres ne correspondent en rien à la structuration avancée par Susan Petit.

59 Le « R » pourrait représenter également la rédemption, ibid., pp. 243-245.
} 
David Gascoigne de son côté se livre également à une analyse de plusieurs romans de Tournier où apparaît son prénom Michel (1996, pp. 207-213). ${ }^{60}$ Bouloumié (1988, pp. 41-54) consacre un chapitre (« Mythe et Nom ») très intéressant à ce qu'elle appelle « l'art de nommer les personnages ». Elle constate que Tournier a une approche « biblique » de l'onomastique:

Pour Michel Tournier, grand lecteur de la Bible, nommer, c'est appeler à l'existence. Le nom est essence, principe d'existence, il engendre l'être ou l'objet qu'il désigne. [...] Michel Tournier redonne au nom sa dimension sacrée.

Bouloumié souligne le rôle de l'étymologie ${ }^{61}$ qui peut résoudre le caractère arbitraire entre le nom et la personne, « comme si le nom pouvait fonder un sens ». D'autre part elle avance le procédé de la réminiscence, ${ }^{62}$ le « nom actualis[ant] un souvenir ». Bouloumié donne les noms de Tiffauges et de Barbe-Bleu comme exemples de cette réminiscence, « clin[s] d'œil au lecteur averti » (1988, p. 44). En d'autres termes, en exploitant ces deux principes Tournier s'efforce d'établir une relation de compréhension et de reconnaissance entre les noms et les personnes: « il attribue [au nom] la tâche d'exprimer l'essence d'un personnage qu'il identifie » (1988, p. 54).

Ce principe de l'onomastique est également signalé par Mariska Koopman-Thurlings, qui utilise le terme d' " emblématique » pour caractériser le choix des noms par Tournier, qui ainsi « renoue avec l'ancienne tradition littéraire » (1995, p. 201). Sa réflexion sur le nom de Tiffauges est trop intéressante pour ne pas la citer in extenso:

[...] Tiffauges [...] connaît la double articulation du réel et de l'irréel. Il appartient au monde de la réalité par sa profession de garagiste et ses occupations quotidiennes, mais il s'inscrit dans un réseau légendaire par son nom ambigu, qui réunit la vocation de victime aussi bien que celle de bourreau. Le nom d'Abel réfère à la victime du fratricide primordial, et le nom de Tiffauges évoque le personnage de Gilles de Rais, qui aurait ${ }^{63}$ violé et tué de nombreux enfants. (1965, p. 202)

Pour W.D. Redfern « [w] ordplay serves both mystification and myth-making or, more strictly remaking » (1985, pp. 304-319). Il constate que beaucoup de mythes sont fondés sur un jeu étymologique, notamment avec des noms. Cependant, l'étymologie ne se limite pas à la seule onomastique, elle est à la base du jeu de

\footnotetext{
60 David Gascoigne titre son épilogue: « Tournier's Signature ».

61 Voir aussi Koopman-Thurlings (1995, pp. 185-188).

62 Ce procédé de la réminiscence est expliqué également par Bouloumié (1993, pp. 9-20). On peut se demander si le plagiat, dont la présence dans l'œuvre de Tournier est reconnue voire revendiquée par luimême, n'est pas la forme extrême de l'intertextualité en tant que réminiscence littéraire.

63 Étant donné que les crimes commis par Gilles de Rais ont été prouvés pendant son procès et suivis de verdict (la pendaison suivie par le bûcher), il n'est pas lieu de l'utilisation du conditionnel qui sème le doute sur le caractère réel des faits.
} 
mots en général chez Tournier, pour qui « l'approche de l'absolu se signale par le rire » $(\mathrm{MTR} 1415){ }^{64}$

Ce qui frappe, c'est que Tiffauges ou le narrateur explique parfois les noms dans le détail et parfois non. Il est donc question dans le roman de noms « clair[s] et distinct[s] » (MTR 421), relevés souvent aussi aisément par la critique, qu'il convient de rappeler ici non pas tant par souci d'intégralité que par la constatation que le dernier mot n'a pas été dit sur certains de ces noms « évidents ». Et il y a donc aussi des noms « difficile[s] à lire », noms qui sont autrement plus intéressants car ils font « approfondi[r] et gagne[r] en richesse » le roman, pour reprendre la parole de Tiffauges (MTR 421).

\section{De l'onomastique explicitée ou plutôt évidente vers les noms « difficiles à lire » ${ }^{65}$}

Dès la deuxième page du roman le protagoniste se présente: «Je m'appelle Abel Tiffauges, je tiens un garage place de la Porte-des-Ternes [...]» (MTR 192). L'origine du prénom Abel, ${ }^{66}$ dont le caractère de nomade est expliqué par le protagoniste luimême, lui va à merveille, tout comme le patronyme Tiffauges. C'est au Professor Doktor Otto Blättchen ${ }^{67}$ qu'incombe l'honneur de déchiffrer ce nom. De par l'ironie le double titre de ce scientifique de la race prête à rire, d'autant plus que le mot allemand Blättchen signifie en français petite feuille (de papier). Il rapproche le nom de Tiffauges de l'allemand «Tiefauge*, c'est l'œil profond, l'œil enfoncé dans l'orbite ${ }^{68}$ (MTR 422), mot qui correspond parfaitement à la physionomie de Tiffauges. Avec l'emploi du mot « sobriquet » il fâche Tiffauges, qui se retient pour exploser cependant plus tard quand Blättchen se permet de faire encore un autre jeu de mots sur son patronyme en l'appelant « Herr Triefauge », jouant ainsi sur l' « œil malade », la myopie de Tiffauges. C'est un des rares moments où Tiffauges se fâche et devient menaçant. Ses yeux qui « ont rempli leurs orbites au point de saillir audehors » (MTR 423) font penser au dessin de l'ogre fait par Gustave Doré pour illustrer Le Petit Poucet (Fig. 2). Jean-Bernard Vray n'exclut pas une forme de rivalité entre Blättchen et Tiffauges, les deux se livrant à des expériences, criminelles pour l'un et perverses pour l'autre. Plus loin Vray constate que « Blättchen et Tiffauges pratiquent tous les deux l'analyse anatomique » (1997, pp. 141 et 183).

\footnotetext{
64 Tournier consacre le passage en question à l'influence de Gaston Bachelard sur lui, et notamment la découverte de l'importance du rire.

65 Force est de reconnaître que cette transparence onomastique varie selon les (connaissances et efforts des) lecteurs.

66 Voir MTR 217, 227 et surtout 437. Jonathan Krell, consacre un chapitre très intéressant à l'opposition entre le sédentarisme et le nomadisme et leur rôle dans l'œuvre de Tournier (1994, pp. 26-31, 103-104). Pour les références bibliques d'Abel, voir Gascoigne (1996, pp. 106-108), et Koster (1995, pp. 136-138).

67 Voir aussi plus loin nos remarques sur les patronymes allemands. Liesbeth Korthals Altes (1992, p. 85) relève aussi les noms significatifs des autres professeurs allemands, Keil (= " goupille ») et Heck (=« enclos, haie »). Ajoutons encore celui du professeur Essig (= « vinaigre »).

68 Dans son article consacré à Tournier et Jonathan Littell, Rasson (2013, pp. 119-128) écrit au sujet des noms des deux protagonistes de leur roman: « Et s'il est licite de voir dans le nom de Aue une déformation du mot allemand Auge, le nom du protagoniste de Tournier est, lui, germanisé en Tiefauge [...], un nom de famille que le personnage des Bienveillantes aurait très bien pu porter ».
} 


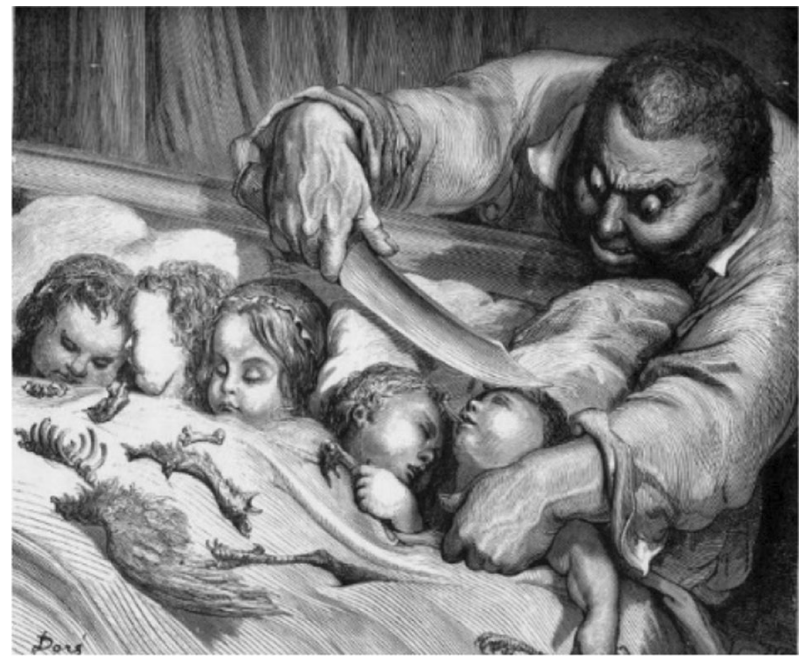

Fig. 2 Gustave Doré, Le Petit Poucet. Wikimedia Commons

« Tiefauge » et « Triefauge »: deux jeux de mots par l'intermédiaire de l'allemand, dont la connaissance approfondie acquise par Tiffauges en quelques années seulement est aussi surprenante qu'invraisemblable. À moins que l'on y voie l'effet du surdéveloppement de son ouïe. Cependant il n'y a rien dans le roman sur cette autre allusion bien évidente: celle à Tiffauges, le nom du château de Gilles de Rais, qui peut être considéré comme archétype de l'ogre. ${ }^{69}$ Pour Jean-Bernard Vray « Gilles de Rais est [...] métonymiquement inclus dans Tiffauges par le choix de ce nom » (1997, p. 126). L'explication du nom du cheval de Tiffauges ne fait pas non plus allusion à Gilles de Rais qui a servi de modèle pour le personnage mythique de Barbe-Bleue $^{70}$ :

Un soir, l'Oberforstmeister revint de Trakehnen en menant au cul de sa charrette anglaise un hongre noir gigantesque, bosselé de muscles, chevelu et fessu comme une femme.

[...]

Un matin que le cheval était touché par un rayon de soleil tombant à contre-jour, il (=Tiffauges) s'avisa que son poil d'un noir de jais présentait des moires bleutées en forme d'auréoles concentriques. Ce barbe était ainsi un barbe bleu, et le nom qu'il convenait de lui donner s'imposait de lui-même. (MTR 388-389)

\footnotetext{
${ }^{69}$ Voir Bataille (1965). Notons aussi le palindrome formé par le patronyme de Gilles de Laval, sire de Rais.

70 Voir à ce sujet notre article écrit en collaboration avec Smith (2009).
} 
On notera que Tiffauges féminise par la suite son «barbe bleu* », à savoir le nom du type de cheval, en « Barbe-Bleue », montrant ainsi sa connaissance du conte de Perrault et réaffirmant par la même occasion sa sensibilité aux jeux de mots. L'apparition répétée du mot hongre ${ }^{71}$ dans ce contexte confirme le rapprochement étymologique et anagrammatique avec ogre ainsi que le microgénitomorphisme de Tiffauges.

Tiffauges avoue sa propension pour le jeu avec les lettres au sujet du mot «inspiration* ${ }^{*}$ :

« Ici, je ne joue pas sur les mots. Il est logique qu'à ce niveau, le sens propre et le sens figuré se confondent, tout comme on ne doit jamais perdre de vue qu'esprit vient de spiritus* dont le premier sens est souffle*, vent* ${ }^{*}$ (MTR 466).

La première phrase de cette citation laisse entendre que le jeu sur les mots lui est familier ailleurs. Cette prédilection du jeune Tiffauges pour le jeu avec le double sens des mots et des lettres avait déjà apparu dans la scène du tatouage, défini comme « tracer profondément des signes sur la peau sans l'écorcher » (MTR 199), au collège Saint-Christophe, union du signe et de la chair. Les « énigmatiques initiales » de la formule «A T pour la vie* » tatouée par Tiffauges sur la cuisse de Pelsenaire peuvent en effet avoir plusieurs significations: «A toi pour la vie* », « Abel Tiffauges pour la vie*» ou encore « Athée pour la vie » (MTR 199-200).

Pour Liesbeth Korthals Altes (1992, p. 59) cette scène montre que « déjà pour Tiffauges enfant l'écriture était le premier acte par lequel il cherchait à s'assujettir l'entourage qui l'opprime ». «A toi pour la vie* » relève de la soumission, « Abel Tiffauges pour la vie* » de la domination. Korthals Altes met cette équivoque en rapport avec les Juifs à Auschwitz « marqués dans leur chair ». Le tatouage de Pelsenaire par Tiffauges semble aussi annoncer les signes de la chair dont il est question dans les fragments où les enfants sont sélectionnés en fonction de leurs caractéristiques aryennes à l'entrée dans les napolas.

Si cette scène montre bien avec quel soin Tournier a choisi le nom et même les initiales du protagoniste de son récit en cherchant à leur donner une motivation linguistique, il en est de même pour la « victime » de Tiffauges. Originaire du nord de la France, de la Belgique et des Pays-Bas, le patronyme de Pelsenaire est à mettre en rapport étymologique avec le nom professionnel de pelseneer, « celui qui travaille la peau » en moyen néerlandais, le verbe « pelsen » signifiant « travailler la peau ». Personnage impressionnant, il est présenté par Tiffauges dans son journal sous ce même aspect « cutané »: «Une bonne part de son prestige tenait à un ceinturon de cuir d'une largeur inouïe - j'ai appris plus tard qu'il avait été taillé dans une sousventrière de cheval » (MTR 198). Nous verrons plus loin qu'avec le choix des noms de quelques autres personnages du roman l'auteur fait preuve de cette même application pour établir un rapport significatif entre patronyme et personnage en réduisant l'arbitraire entre les deux.

\footnotetext{
71 Le Petit Robert 2016 définit « hongre » comme « Châtré, parlant du cheval ». D'origine hongroise, les mots « hongre » et « ogre » sont étymologiquement apparentés (Krell 1994, pp. 92-93).
} 
Le nom du collège Saint-Christophe est expliqué in extenso dans le journal de Tiffauges, qui termine ces pages avec les termes « géant Porte-Christ » (MTR 227). Par le choix de ce nom du collège Tournier détermine ainsi de manière claire et nette le cadre judéo-chrétien ${ }^{72}$ de ce roman mythologique tout en l'inscrivant dans le thème omniprésent de la phorie. En revanche, aucune explication pour la Portedes-Ternes où se trouve le garage de Tiffauges. Pourtant il n'est pas moins éloquent: le thème phorique y apparaît textuellement. De plus, Tiffauges constate que son « métier de garagiste [le] replace sous le patronage du géant Porte-Christ...» $(\mathrm{MTR} 217) \mathrm{du}$ fait qu'il « entretien[t] et répare cet instrument par excellence de la migration, l'automobile » (MTR 218). De toutes les Portes de Paris que l'auteur aurait pu choisir, il a bien sûr retenu la Porte-des-Ternes: le sens du mot terne ${ }^{73}$ se rapproche de celui de sinistre et correspond le mieux à l'ambiance lugubre du roman et de son époque. Signalons dans ce contexte le nom de Cromorne, protagoniste du roman commencé et abandonné par Tournier en $1958 .{ }^{74}$ Précurseur de Tiffauges, ce Cromorne est garagiste comme lui. Tournier a déclaré avoir choisi le nom de Cromorne, qui devait avoir un sens en allemand et en français, pour sa consonance belle et triste. En plus le nom de Cromorne est celui d'un instrument de musique, le Krummhorn en allemand. Tournier lui-même appelle Les Plaisirs et les Pleurs d'Olivier Cromorne « une prémonition du Roi des Aulnes. Olivier Cromorne, c'est Abel Tiffauges avant la guerre », ou encore « une première mouture du Roi des Aulnes » $\left(2011\right.$, pp. 84-85, 90, 93).$^{75}$ À Cromorne il a préféré cependant le nom de Tiffauges, jugé plus riche vu son rapport avec le château de Gilles de Rais et avec les mots allemands Tief Auge. ${ }^{76}$

Les arguments qui sont à la base du choix de la Porte-des-Ternes à Paris se retrouvent encore dans celui de la banlieue parisienne de Pantin où se trouvent les origines de Phiphi, le plus jeune prisonnier de guerre de la baraque où est interné Tiffauges dans les environs de Moorhof ${ }^{77}$ en Allemagne. «Personne comique ou ridicule par ces gesticulations excessives ${ }^{78} »$ : le nom de « pantin » convient parfaitement à ce Phiphi « qui fatiguait tout le monde de ses calembours et de ses grimaces » (MTR 337). Quand on prend en considération que la caractéristique principale du calembour est le double sens, on est frappé par le procédé du dédoublement utilisé par le narrateur dans les passages consacrés à ce personnage malheureux. D’abord, bien sûr, dans le diminutif de son nom Phiphi, puis dans le double sens de «Pantin/pantin » et les

\footnotetext{
72 Avec le déplacement de Tiffauges en Allemagne, le cadre parallèle germanique devient de plus en plus important.

73 L'adjectif « terne » dans le sens de « sans éclats, délavé, fade » correspond également aux photos ou images en noir et blanc de la période de la Seconde Guerre mondiale vues par les yeux du spectateur de l'époque après qui est habitué aux clichés et aux vidéos en couleur. David Bevan relève aussi le choix judicieux de ce toponyme (1986, p. 34).

${ }^{74}$ Arlette Bouloumié en parle au sujet des manuscrits de Tournier dans la « Note sur la présente édition » qui figure dans Tournier (2017, MTR LII-LIII).

75 Arlette Bouloumié avance que « [1] e roman Les Plaisirs et les pleurs d'Olivier Cromorne [...] était très noir. Les Ecrits sinistres qui en sont inspirés en gardent un souvenir atténué » (2011, p. 245).

76 Voir à ce sujet Bouloumié, (2014, p. 127). Le nom de Cromorne a été retenu par Tournier pour réapparaître dans le conte Tupik dans Le Coq de bruyère.

77 Moorhof signifie « cour de marécage » et annonce ainsi la fin du roman.

78 Définition de « pantin » donnée par Le Petit Robert 2016.
} 
multiples significations « grimaces $» .{ }^{79}$ Contrarié par ces camarades, Phiphi «s[e] défendit par un feu d'artifice d'à-peu-près [...] ». Le premier terme de cette citation dédouble la feinte de la grimace, le second est synonyme de calembour. Phiphi finit par se suicider pour des raisons qui restent inexpliquées, « on le retrouva mort, pendu à un poteau de l'enceinte avec sa ceinture » (MTR 345). Tout compte fait, le narrateur a recours dans ses descriptions de Phiphi à la même propension que celle qui caractérise Phiphi, ce qui nous amène à penser que Tournier pourrait très bien se moquer ici de lui-même, lui qui aime tout autant les jeux de mots et les calembours, sachant d'ailleurs et confirmant ainsi qu'ils ne sont pas forcément efficaces. ${ }^{80}$ Le jeu langagier auquel se livre Tournier constitue une partie intégrante de son humour ou de son côté insolite, sans qu'il ne perde jamais de vue l'objectif qu'il cherche à atteindre et communiquer. Cette conception littéraire de Tournier a été très bien exposée par Susanna Alessandrelli (2013, p. 48):

L'humoriste exploite de préférence l'ambiguïté des rapports entre signifiant et signifié, ou brouille délibérément les règles qui les régissent. Toutefois, chez Michel Tournier, les jeux avec le langage sont toujours loin d'être innocentes et sont plutôt au service d'un usage subversif du message littéraire.

Tel qu'il nous est décrit, il semble qu'à la différence de Tournier, l'usage des calembours par Phiphi est gratuit et donc condamné à échouer. ${ }^{81}$ Le rapprochement avec le concept du « diabole », qui est symbole vidé de sens, s'impose. Le suicide de Phiphi serait ainsi apparenté à l'autodestruction du régime nazi annoncée par le comte de Kaltenborn. À moins que la mort de Phiphi soit justifiée par le manque de respect voire la ridiculisation de la langue, allemande en l'occurrence, des mots et des noms:

Il s[e] défendit par un feu d'artifice d'à-peu-près où entraient pêle-mêle les noms des uns et des autres, ceux des rues et des bistrots de Pantin et les mots tudesques - grotesquement ${ }^{82}$ francisés - qu'il avait glanés depuis le début de sa captivité. (MTR 345)

Tiffauges essaie de surmonter son désespoir moral en se livrant à la pratique du «brame* ${ }^{*}$, espèce de rite où « s'exhale tout l'ennui de vivre et toute l'angoisse de mourir» (MTR 227). Cette pratique, qui montre au plus fort sa folie, lui est venue par la lecture du roman Le Piège d'or de James Oliver Curwood que lui a prêté Nestor. Le héros de ce récit, qui se déroule au Canada, c'est Bram Johnson, « un colosse sauvage [pour qui] hurler avec les loups n'était pas une figure de style » (MTR 222). Par l'intermédiaire d'un jeu de mot avec le nom anglais du protagoniste

\footnotetext{
79 « Grimace »: (1) « expression caricaturale du visage »; (2) « attitude maussade de personne mécontente »; (3) « mine affectée par feinte » et (4) « figure grotesque » (Source Le Petit Robert 2016).

80 Voir aussi à ce sujet Redfern (1996, pp. 112-113).

81 Une certaine prudence s'impose ici. Avec Le pied de la lettre, Trois cents mots propres, Tournier semble bien avoir voulu exprimer et concrétiser son amour pour les mots en soi. L'attirance pour lui des mots en « $\mathrm{k} »$ en est un bel exemple.

82 Avec sa propension au grotesque, Tournier se rapproche là encore de la conduite de Phiphi. Voir pour le rôle du grotesque chez Tournier: Korthals Altes (1993, pp. 77-91).
} 
de ce roman, « le cri de Bram » devient le «brame* » de Tiffauges. Le brame des cerfs en rut est encore évoqué par Tiffauges quand il est question du magnétophone de Karl F., dont le fonctionnement au niveau du son se compare parfaitement à celui de l'appareil photo par rapport à l'image ou encore à celui de l'écriture par rapport au papier. Plus tard, depuis sa cabane appelée le « Canada » (MTR 346), ${ }^{83}$ Tiffauges entend le brame naturel des cerfs, notamment celui de l' «Unhold* », qui est « si profond qu'on aurait dit le rire d'un géant ventriloque » (MTR 353). Dans ce contexte de brames et de cris incontrôlés il convient de signaler aussi les sens du mot « unhold » en anglais: « disgracieux/repoussant » et « relâcher/laisser échapper », ce qui correspond entièrement à la pratique à laquelle se livre Tiffauges. Que Tournier n'hésite pas à recourir aux langues étrangères pour ses jeux onomastiques nous est révélé aussi par Dalmas (2005, p. 99), qui signale que l'orthographe du nom Helmut est changée en Hellmut, sous l'influence de l'anglais « enfer ». ${ }^{84}$

Tiffauges se déclare très sensible à la poésie musicale des prénoms allemands, qui exercent sur lui un véritable enchantement. La description qu'il fait « de la belle et longue litanie de l'appel » (MTR 480) des quatre cents garçons de la napola en témoigne $^{85}$ :

Il n'est pas de plus douce musique pour moi que ces prénoms évocateurs, criés par des voix toujours nouvelles et sur lesquelles se posera à son tour le prénom qui lui revient. Ottmar aus Johannisburg, Ulrich aus Dirntal, Armin aus Königsberg, Iring aus Marienburg, Wolfram aus Preussisch Eylau, Jürgen aus Tilsit, Gero aus Labiau, Lothar aus Bärenwinkel, Gerhard aus Hohensalzburg, Adalbert aus Heimfelden, Holger aus Nordenburg, Ortwin aus Hohenstein...

\footnotetext{
${ }^{83}$ Nous ne reviendrons pas sur l'importance du Canada pour Tiffauges, celle-ci ayant été analysée de manière exhaustive et convaincante par Vray (1997). Vray distingue trois phases dans le cycle canadien du Roi des Aulnes (pp. 34-41) et analyse le caractère mythique du Canada (pp. 425-429). Voir aussi Bergholz (2010, pp. 111-131), Bevan (1986, p. 25), et deux textes de Platten (1991, pp. 287-290 et 1999b, pp. 87-89). Ajoutons seulement aux trois apparitions du Canada (le roman de Curwood, la cabane en Prusse-Orientale, le dépôt des possessions des Juifs dans les camps d'extermination) qu'on peut se demander si Tournier ne s'est pas laissé inspirer par le tableau dressé dans la première partie de Ma cabane au Canada, chanson « utopique » de Line Renaud. Citons trois vers de cette chanson qui faisait fureur vers la fin des années 40 et où on retrouve le cadre de l'abri de Tiffauges, y compris l'élan: « Ma cabane au Canada, c'est le seul bonheur pour moi », « Toujours l'élan de mon cœur/Reviendra vers ma cabane au Canada ». D'un autre registre, mais tout aussi curieux est l'apparition du nom de Bob Watson, le mari d'Édith, ancienne chanteuse d'opéra dans Le nain rouge. Il pourrait très bien être inspiré par le Bobby Watson de La cantatrice chauve d'Eugène Ionesco.

${ }^{84}$ Hellmut von Bibersee est un des Jungmannen de la napola décapité par la flamme arrière d'un Panzerfaust (MTR 495-497). Ce fragment ressemble étrangement à une scène du film Die Brücke (Le Pont 1959) du metteur en scène Bernhard Wicki, où des lycéens sont tués dans les mêmes circonstances en avril 1945. À l'approche de l'armée américaine, ces membres mobilisés dans le Volkssturm, âgés de 16 ans et totalement sans expérience militaire, sont appelés à défendre un pont devenu stratégiquement inutile. Ce film, qui montre l'endoctrinement de la jeunesse allemande par le national-socialisme, fut un des plus grands succès du cinéma allemand d'après-guerre. Comme il l'a déclaré dans les Lettres parlées à son ami allemand Hellmut Waller, Tournier (2015) a admiré ce film, dont on reconnaît plusieurs éléments dans les dernières pages du roman.

${ }^{85}$ Ce faisant, Tiffauges est évidemment le porte-parole de Tournier qui, lui aussi, s'émerveille devant la beauté des noms (voir supra). Cette litanie contraste fortement avec l'énumération des noms dont il est question dans le fragment de Phiphi de Pantin.
} 
Je dois me faire violence pour interrompre ce recensement de mes richesses [...] (MTR 480)

Quelle différence avec cette autre énumération de la « carte géographique infernale »:

Schirmeck, Natzviller, Dachau, Neuengamme, Bergen-Belsen, Buchenwald, Oranienburg, Theresienstadt, Mauthausen, Stutthof, Lodz, Ravensbrück... Ces noms avaient dans la bouche d'Éphraïm la valeur de points de repère familiers sur cette terre des ombres qui était la seule qu'il connût. Mais aucun ne brillait d'un éclat aussi noir que celui d'Oswiecim, à trente kilomètres au sud-est de Katowice, en Pologne, que les Allemands appelaient Auschwitz. (MTR 505)

Les nombreux noms géographiques allemands qui figurent dans les chapitres III à VI paraissent brosser essentiellement la couleur locale de l'Allemagne et notamment de la Prusse-Orientale où se déroulent ces parties du roman. Plus qu'un certain exotisme, ils semblent encore évoquer le déclin de cet empire allemand, cette connotation étant bien sûr aussi le résultat des connaissances historiques du lecteur d'après-guerre. Certains noms allemands sont connotatifs, allusifs ou associatifs pour les germanophones: Raufeisen évoque en même temps le « rapt », le « vol », le « froid », le « fer » et l' « épée », correspondant ainsi parfaitement à sa fonction et sa conduite dans la napola de Kaltenborn, dont la première syllabe du nom reprend précisément ce caractère froid et insensible de l'éducation et de la discipline de fer imposées aux futurs SS. Dans Le Vent Paraclet (MTR 1383) Tournier déclare n'avoir jamais mis les pieds en Prusse-Orientale, la description de cette région est donc purement fictive, certes après documentation sérieuse. Avec l'invention du nom de Kaltenborn Tournier fait preuve de ce qu'on pourrait appeler une « intuition onomastique »: il apprend qu'après la guerre le dernier conservateur de la réserve de Rominten « s'était replié en Allemagne de l'Ouest et administrait une réserve de chasse [...] près de Kaltenbronn [...] Je fus assez impressionné par la similitude de ce nom de localité réelle avec celui de la forteresse imaginaire de Kaltenborn où j'avais logé ma napola en Prusse-Orientale » (Le Vent Paraclet, MTR 1388). Une variante de cette intuition est l'esprit prémonitoire de Tournier, qu'il révèle également dans Le Vent Paraclet (MTR 1403) en racontant sa découverte de la PrusseOrientale en 1975, donc bien après la parution du roman: « [...] j'admirais qu'ayant à découvrir la Prusse-Orientale ce fût en compagnie d'un géant amateur de lait et d'enfants (=Philippe Janssen), tel exactement que j'avais imaginé Abel Tiffauges ». Voilà l'imaginaire romanesque rattrapé après coup par la réalité...

Les éminences scientifiques nazies (Essig, Keil, Heck et Blättchen ${ }^{86}$ ) sont plutôt ridiculisées, aussi bien par leurs patronymes que par leurs théories et pratiques contestables et par les titres académiques pompeux et grotesques dont ils sont dotés. Il ne va pas de même pour le professeur Unruh, « savant généticien » (MTR 317), qui

86 Voir aussi note 68. 
avait réussi à reproduire des pigeons qui sont des jumeaux artificiels. ${ }^{87}$ Les travaux génétiques du professeur docteur Lutz Heck, « père de Bos Primigenius Redivivus », dont il est question avec beaucoup d'ironie dans la partie L'Ogre de Rominten (MTR 371), font écho à ceux du professeur Unruh sur les pigeons. Ils sont les précurseurs animaliers des expériences médicales livrées par le Dr Mengele sur les humains, et notamment sur la gémellité. Pendant ses rafles de pigeons, qui annoncent celles des enfants pour la napola de Kaltenborn, Tiffauges rencontre la veuve du professeur qui s'inquiète qu'il lui prenne le pigeon argenté, « ce qu'[elle a] de plus cher au monde depuis la mort du professeur ». Rien à faire, par l'attitude imperturbable et implacable de Tiffauges, «[e]lle comprit alors que si le pigeon platiné était un symbole pour elle, il était bien davantage encore pour Tiffauges » (MTR 220). Or, « le visage crayeux et les lèvres tremblantes de Mme Unruh » correspondent à merveille à son patronyme qui signifie inquiétude en français.

Une des scènes les plus horribles du roman est sans doute la mort d'Arnim, le garçon de la napola qui est pulvérisé par la mine (« lourd disque de mort » (MTR 499), comme un Atlas déchu) qu'il porte. Arnim, porteur de mine jusque dans son nom. Par la puissance de l'explosion Tiffauges est jeté à terre et couvert par le sang du garçon. Dans ses Écrits sinistres il décrit cet événement avec un lyrisme religieux, ${ }^{88}$ faisant allusion directe à la conversion de Saul à Damas. Le prénom d'Arnim préfigure et inclut cette explosion par la mine et par l'inversion des trois lettres il mime le renversement littéral et figuré de Tiffauges. ${ }^{89}$

Le nom du dernier guillotiné en public en France, Weidmann, présente également un intérêt particulier pour le roman. Cet assassin, qui ressemble comme deux gouttes d'eau à Tiffauges, est né le même jour que lui. Ayant fait le chemin inverse de Tiffauges en quittant l'Allemagne pour la France, « le géant aux sept crimes » (MTR 295) a été condamné à mort pour avoir tué sept personnes et est exécuté ${ }^{90}$ le 17 juin 1939 sous les acclamations d'une « foule hargneuse » (MTR 297). Avec son nom qui signifie chasseur en français, il s'inscrit ainsi dans plusieurs grands thèmes

\footnotetext{
87 Ces pigeons intègrent ainsi le thème de la gémellité élaboré dans les détails au sujet de Haïo et de Haro, « jumeaux-miroirs* » (MTR 448). Sans doute Tournier aurait été très intéressé par les récentes recherches de la NASA sur les jumeaux Kelly, qui ont été séparés pendant plus d'un an, Scott séjournant dans l'espace et Mark sur terre. Le vieillissement du dernier par rapport à son frère permettrait de conclure à l'existence d'une source de jouvence dans l'espace. Ces deux thèmes, la gémellité et la jouvence, réunies ici scientifiquement, jouent un rôle important dans l'œuvre de Tournier, qui se sert du terme de « juvénophilie » pour indiquer que « l'une des caractéristiques du fascisme [est] de surévaluer la jeunesse » (Le Vent Paraclet, MTR 1383-1384).

88 Pour Liesbeth Korthals Altes (1993, p. 89) ce « passage présente un sacré ambigu, ambiguïté qui tient à l'encodage d'une double perspective: pour le personnage, la scène est grave et relève d'un sacré authentique. Pour le lecteur, le détournement de formes et de contenus religieux traditionnels est flagrant, sans que toutefois se perde la dimension sacrale. Le grotesque est d'un sacré à la fois intense et sacrilège $\gg$.

${ }^{89}$ Le choix de ce prénom d'Arnim et non pas du prénom usuel et beaucoup plus répandu d'Armin semble confirmer cette inversion qui reflète le renversement.

90 C'est la dernière exécution publique en France.
} 
du roman à la fois: la chasse, la gémellité, ${ }^{91}$ l'inversion, la peur et le pressentiment du rejet par la foule ainsi que la mythologie exploitant le chiffre sept. ${ }^{92}$

\section{L'onomastique « difficile à lire »: des chiffres et des lettres}

Par la difficulté à lire l'auteur pose des exigences au niveau de la compréhension et de l'interprétation de la part du lecteur. Bevan (1986, p. 70) insiste sur l'importance pour Tournier de « la participation créatrice du lecteur ». Il cite Tournier dans un article paru dans Le Magazine littéraire (1981): « [...] une œuvre naît quand un livre est lu, et [...] cette œuvre est un mélange inextricable du livre écrit, c'est-àdire de la volonté de l'auteur, et des fantasmes, des aspirations, des goûts, de toute l'infrastructure intellectuelle et sentimentale du lecteur. Un livre a toujours deux auteurs: celui qui l'écrit et celui qui le lit». Bevan en conclut: « Rendre plus opaque, rendre plus résistant à une conclusion définitive, c'est exiger du lecteur un plus grand effort, une plus grande participation » (1986, p. 70).

Dans ses Écrits sinistres du 28 mars 1938 Tiffauges se dit accoutumé aux « coïncidences inexplicables dont j'ai pris mon parti comme d'autant de petits rappels à l'ordre* » (MTR 246). Il en donne un bel exemple où le jeu de mots avec ce qu'il appelle des « rappels à l'ordre » est évident:

Il y a six mois, ayant des échéances difficiles, j'ai acheté un billet entier de la Loterie nationale en prononçant cette courte prière: « Nestor, pour une fois ? » Oh, je ne peux pas dire qu'on ne m'a pas entendu ! On m'a même répondu. Par une manière de pied de nez. Mon numéro était le B 953 716. Le numéro qui a rapporté un million à son propriétaire était le B 617 359. Mon numéro à l'envers. C'était pour m'apprendre à vouloir tirer un profit trivial de ma relation privilégiée avec le ressort de l'univers. Je me suis fâché, puis j'ai ri. (MTR 246)

Ce fragment est intéressant de plusieurs points de vue. D'abord, le billet de loterie acheté par Tiffauges pour gagner un ballon d'oxygène et renflouer la caisse de son garage situé près du Ballon des Ternes (MTR 248), sculpture de Bartholdi, porte très exactement deux séries de trois chiffres, soit deux ternes précédés de la lettre B. Ce Ballon des Ternes est en effet l'œuvre de Bartholdi, le créateur de la statue de la Liberté et du Lion de Belfort. Sur la page Wikipedia Porte-des-Ternes on trouve la description suivante:

Le groupe représentait la Ville de Paris, sur les genoux de laquelle reposait un enfant tendant les mains vers un pigeon lui apportant des nouvelles du pays. Auprès d'eux se tenaient un jeune homme armé d'une épée et un marin se

\footnotetext{
91 Les noms de Haro et Haïo s'inscrivent également dans le thème de la gémellité, la minime différence entre l'un et l'autre s'y reflète. Tout comme les noms de Bidoche et de Bodruche dans Que ma joie demeure (Tournier 1980). Ces noms de jumeaux, vrais ou faux, présentent la même assonance, leurs doubles syllabes se ressemblent et répètent encore le binaire des personnages.

92 Par exemple Petit Poucet, porteur des bottes de sept lieues, qui a sept ans, et ses six frères ou les sept petites filles de l'ogre dans le conte du même nom.
} 


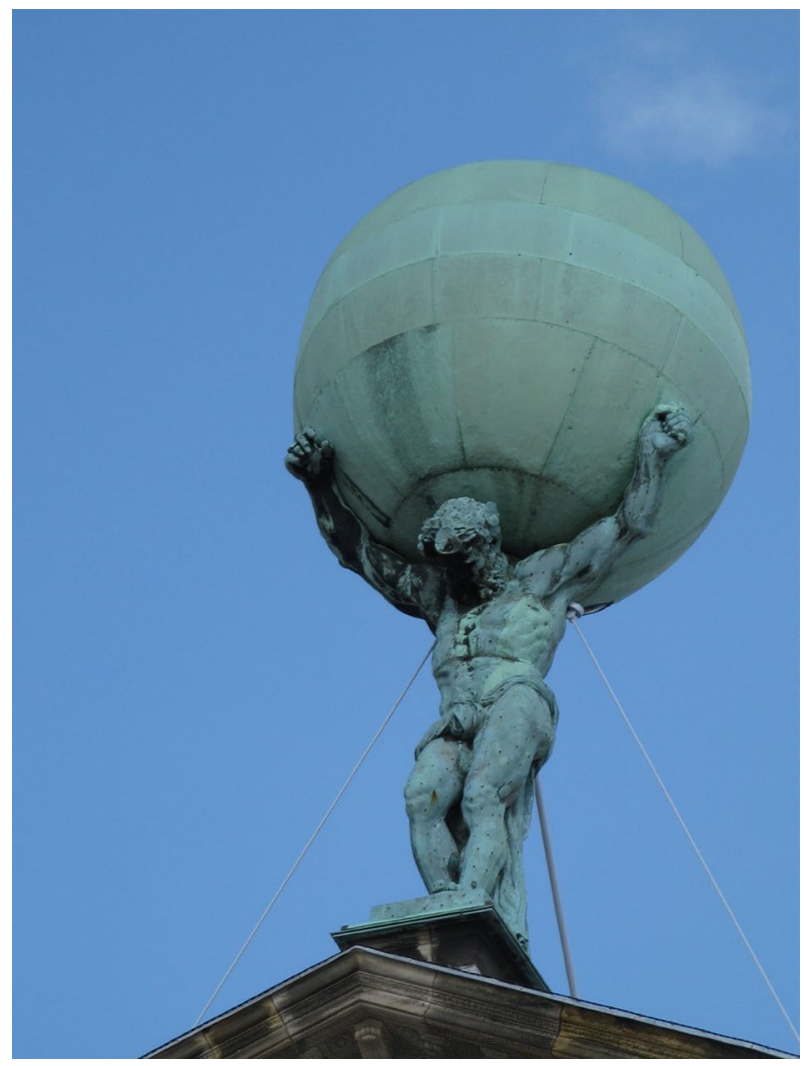

Fig. 3 Artus Quellinus, Atlas (Palais royal Amsterdam). Wikimedia Commons

cramponnant à l'aérostat. Un imposant ballon de cuivre martelé couronnait l'ensemble de ce groupe commémoratif. Il fut inauguré le 28 janvier 1906 [et] fondu par les Allemands pendant la Seconde Guerre mondiale. ${ }^{93}$

L'enfant qui tend la main vers un pigeon « collé par un bout de l'aile » (Queneau 1980) n'a pas dû passer inaperçu pour Tournier, qui a habité Neuilly à partir de 1941 et fréquenté le lycée Pasteur de Neuilly, situé à deux pas de la Porte-des-Ternes. À première vue les aéronautes représentés sur le socle de la statue semblent porter le ballon, à la réflexion on se rend compte que c'est tout le contraire, bien sûr. Autre inversion: cette statue de ballon céleste fait penser à l'image connue d'Atlas portant le globe terrestre, inversion explicitée par Tiffauges comme suit: «Je m'avise en feuilletant un dictionnaire qu'Atlas portait sur ses épaules - non pas le monde, ni la terre comme on le représente habituellement - mais le ciel » (MTR 264). Les Figures 3 et 4 illustrent cette inversion.

93 Source Wikipedia consultée le 15-3-2017 https://fr.wikipedia.org/wiki/Porte_des_Ternes. 
Fig. 4 Frédéric Auguste Bartholdi, Le Ballon des Ternes. Wikimedia Commons

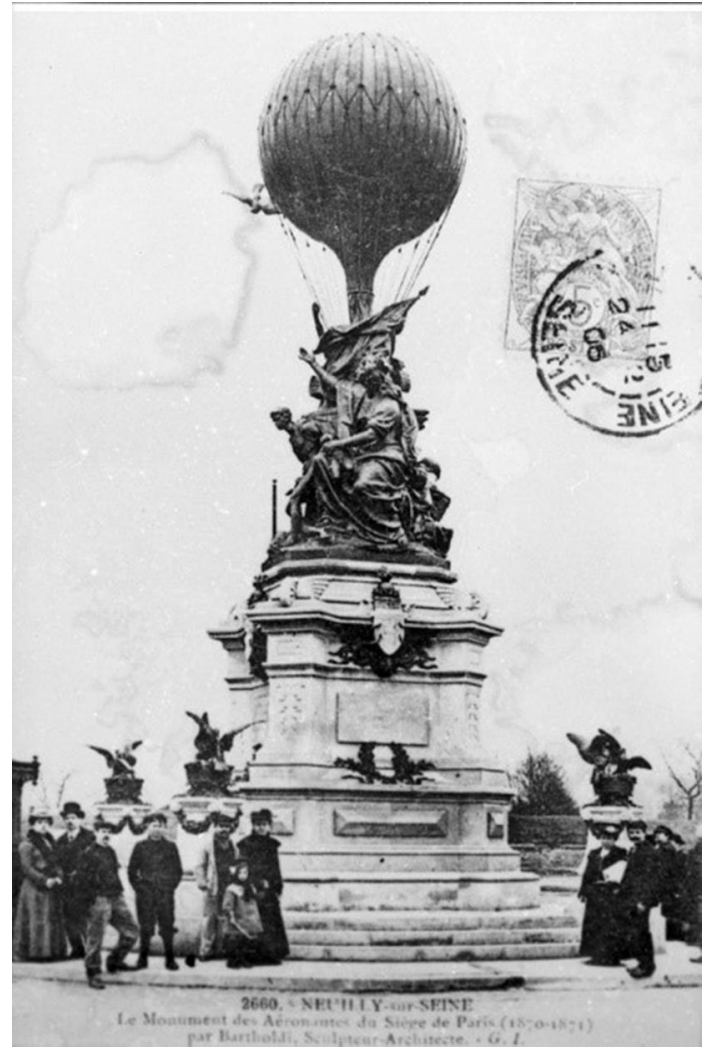

Pour ce qui est du choix de la lettre $B$, rien ne permet de faire avec certitude le rapprochement avec $B$ artholdi et Ballon des Ternes. Cette lettre revient dans le « quartier $B$, où le Dr Mengele se livrait à ses expériences médicales sur les détenus. Mengele [...] s'intéressait passionnément à la gémellité [...] »(MTR 508-509). Dans le même camp de concentration, Auschwitz, débutent en 1941 les premières expériences avec le pesticide Zyklon $B$, ce gaz toxique et asphyxiant est utilisé ensuite à grande échelle pour l'extermination des Juifs. On retrouve la lettre $\mathrm{B}$ encore dans le nom hébreu et biblique de Béhémoth, ici dans le sens de Cheval d'Israël, nom que donne Éphraïm à Tiffauges qui le porte sur ses épaules (MTR $512-513)$. La première description faite par Tiffauges de son garage situé aux portes de Paris est franchement négative avec son « ciel humide et noir », son « odeur de vieille graisse » et son « atmosphère que je hais » (MTR 192). Il lui donne donc aussi des soucis financiers. Il aspire à « échapper à ce garage, aux médiocres occupations [...] et à [lui]-même » (MTR 192), bref de quitter toute cette vie marginale et précaire menée près du faubourg que l'on appelle depuis les années soixante-dix ${ }^{94} l a$ zone. Le sens du verbe zoner couvre aussi toute l'errance désespérée de Tiffauges,

94 Rappelons que Le Roi des Aulnes a été publié en 1970. 
son existence misérable et ses efforts pour y échapper, lui qui, rejeté par la société, cherche «sa » place partout et qui se rend compte de son erreur d'appréciation quand il pense l'avoir trouvée en Prusse-Orientale. Serait-il trop osé de soupçonner ici une lecture alternative du titre du roman, à savoir Le Roi des Zones, ${ }^{95}$ en référence aux zones d'ombre où évolue le protagoniste et à cette précarité sociale, géographique et morale dont Tiffauges se croit et se déclare à la fois victime et champion? Dans Le Vent Paraclet Tournier signale que « [1] ongtemps les Allemands de l'Ouest n'ont consenti à appeler l'Allemagne de l'Est que la 'Zone' (sous-entendu: d'occupation soviétique*) »(MTR 1411). Rappelons que la Prusse-Orientale où sévit Tiffauges avait été «annexée» également par l'Union soviétique après la Seconde Guerre mondiale suite aux conférences de Potsdam et de Yalta.

Cette lecture alternative du titre dédouble celle que l'on reconnaît aisément comme inspirée de la ballade de Goethe, mise en musique par Schubert. L'identification de Tiffauges avec l'homme des tourbières « exhumé [...] dans un petit bois d'aulnes » (MTR 358) est d'abord physique, puis morale vers la fin roman quand Tiffauges va jusqu'à évoquer « [s]a dignité de Roi des Aulnes » (MTR 500). La pratique du rapt d'enfants et l'élément chevaleresque de la ballade se reconnaissent également facilement dans le roman. Dans Le Vent Paraclet Tournier s'explique in extenso sur le rôle, l'origine et l'interprétation de la ballade de Goethe:

Ce poème $[\ldots]$ a toujours été pour l'écolier français $[\ldots] l e^{*}$ poème allemand par excellence, le symbole même de l'Allemagne. Le plus étrange c'est qu'à l'origine de ce poème se trouve une erreur de traduction de Herder qui popularisa le folklore danois en Allemagne. Eller*, les elfes, devint sous sa plume Erlen*, les aulnes [...]. Or il est peu probable que Goethe se fût intéressé à la légende du banal roi des Elfes. En revanche son imagination s'enflamma à l'évocation si précise et originale de l'aulne, parce que l'aulne est l'arbre noir et maléfique des eaux mortes [...]. L'aulne des marécages évoque les plaines brumeuses et les terres mouvantes du Nord, et l'Erlkönig l'ogre aérien, amateur d'enfants, qui plane sur ces tristes contrées. (MTR 1393)

Toute la thématique du roman est ainsi donnée, y compris le décor. Qui plus est, l'erreur de traduction de l'expression folklorique danoise par Herder semble annoncer celle commise par Tiffauges au niveau de l'interprétation de la symbolique nazie.

Dans le contexte du titre du roman ${ }^{96}$ nous aimons revenir à l'article sur les jeux de mots de Redfern, où il parle de l'éventuel plagiat commis par Tournier dans la description du combat chevaleresque entre les garçons au Collège Saint-Christophe

\footnotetext{
95 En parlant du Roi des Aulnes à ceux qui ne connaissent pas le roman, nous avons constaté à plusieurs reprises que c'est de prime abord dans le sens de « Roi des zones » qu'est compris le titre.

96 Didier Decoin trouve que « Tournier est formidablement doué pour les titres » (Tournier 2011, pp. 233-234). Dans une interview avec Franz-Olivier Giesbert Tournier se vante d'avoir choisi de beaux titres pour ses romans et se plaint en même temps que les auteurs ne font pas assez d'effort pour en trouver qui conviennent (Les légendes du siècle, Le Point culture, 5/11/2010).
} 
(MTR 228-230), fragment qui semble en effet être inspirée par une scène du Grand Meaulnes d'Alain-Fournier. Pour Redfern (1985, p. 315), Tournier se serait plutôt livré à une forme d'intertextualité fondée sur ce qu'il appelle « phonic memory »: Tournier/Fournier; aulnes/Meaulnes. ${ }^{97}$ Il convient en effet de ne pas méconnaître le rôle important de l'association formelle dans le processus créatif qui est à la base de l'œuvre de Tournier.

L'ordre des chiffres inversés du billet de loterie reflète aussi l'écriture à reculons du roman. Tournier a indiqué lui-même qu'il construit de préférence ses récits à partir de leur issue ou leur dénouement, en remontant dans le temps narratif: «L'un des secrets consiste à écrire la fin du roman avant le début... Le livre se compose toujours de deux versants... Pour obtenir les correspondances, il suffit de travailler simultanément à chacun des versants. Je n'hésite pas, s'il le faut, à écrire à reculons ». ${ }^{98}$ Dans Le Roi des Aulnes cette technique est très apparente, tout (événements, réflexions, symboles, etc.) converge vers la fin du roman. Écriture à rebours, mais aussi lecture à rebours ${ }^{99}$ : le lecteur (ou encore le chercheur!) n'est-il pas saisi par l'envie de reprendre, de relire le roman depuis le début dès la lecture de la dernière page ? Cette connivence entre auteur et lecteur semble bien être une caractéristique (recherchée) essentielle de l'œuvre de Tournier, pour qui « [...] la lecture est créatrice. Tout lecteur est un créateur $» .100$

Enfin le thème de l'inversion illustré ici au niveau évident des chiffres est un bel exemple de l'humour de Tournier. Dans Le Vent Paraclet (MTR 1391) il écrit que « toute œuvre grande et profonde » se signale par l'humour. Il se moque de sa propre technique d'écriture à rebours dont il est question plus haut, tout en inscrivant cette moquerie dans le thème omniprésent de l'inversion. En revanche, l'humour que fait transparaître Tiffauges est bien limité, les passages où il rit (jaune quand même...) comme dans le fragment précité sont très rares dans le roman, et quasiment absents depuis sa rupture avec Rachel. Notons aussi qu'avant de rire Tiffauges se fâche: ces deux attitudes physiques antithétiques, sans transition, sont bel et bien l'expression du même caractère. ${ }^{101}$ Or, le jeu que l'auteur présente dans ce passage clair et net avec ces numéros/chiffres ${ }^{102}$ se joue également, voire encore plus,

\footnotetext{
97 Notons que le frère du grand-père du côté maternel de Tournier s'appelait également Fournier. Dans Le Vent Paraclet, MTR 1359, Tournier indique que la source germanique de sa famille remonte à ce prêtre et professeur d'allemand. Quelques pages plus loin (MTR 1368) ce patronyme revient quand Tournier parle de ses séjours à Lusigny-sur-Ouche pendant la guerre et de la déportation en 1944 des membres de la famille Fournier, qu'il appelle « ma » famille.

98 Le Monde, 24/11/70. Voir aussi Bouloumié (1988, p. 80). Pour elle, le passage du Rhin constitue la séparation de ces deux versants. Elle constate aussi le rôle important des chiffres et leur symétrie dans le roman en remarquant que la rencontre avec le Roi des Aulnes se situe exactement au milieu du livre, p. 290 sur 580 pages de l'édition Folio.

99 Il n'est pas étonnant que Tournier fût un grand admirateur du naturaliste mythique J.-K. Huysmans, auteur d'À Rebours. Voir Bouloumié (1989, p. 148), et Petit (1986, p. 233).

100 Bouloumié, ibid., p. 149. Voir aussi à ce sujet Davis (1991, pp. 191-206). Magnan (1991, p. 208), utilise le terme de cocréation pour indiquer le rôle du lecteur attendu par Tournier.

101 L'inversion, encore et toujours!

102 Les chiffres dont il est question ici sont en effet moins sujets à discussion que ceux de l'énumération des conquêtes de Don Giovanni par Tiffauges (MTR 273), chiffres dont la différence avec le libretto de Mozart est analysée de manière convaincante par Jiménez (2016, pp. 329-336).
} 
au niveau des lettres. Ainsi Redfern constate que « Tournier's wordplaying is itself 'une inversion maligne'- a clever, controlled, often malicious twist » (1985, p. 313). Worton abonde dans ce sens en relevant le choix par Tournier d'introduire dans son conte La Reine blonde un certain Edward Reinroth: «Édouard étant le deuxième prénom du romancier et Reinroth la quasi-anagramme de Tournier, on peut penser qu'il s'inscrit lui-même dans son conte, de façon subversive et ironique [...] » (1991, p. 239). ${ }^{103}$ Nous ajouterions que la signification ( « rouge pur »), la connotation germanique et la référence géographique du pseudonyme inversé de Reinroth ne sont pas non plus à négliger.

Le choix d'un grand nombre de noms dans le roman nous semble en effet confirmer que Tournier s'est livré dans ce domaine encore à un travail de création, mieux de bricolage, en forgeant non pas des noms relativement faciles à comprendre, comme nous l'avons vu pour celui d'Abel Tiffauges, mais plutôt énigmatiques et difficiles à déchiffrer par leur forme anagrammatique ou leur caractère connotatif.

\section{Rachel et Éphraïm}

Le nom de Rachel est le premier à paraître dans les Écrits sinistres (MTR 191). C'est aussi elle qui est la première à reconnaître le caractère d'ogre de Tiffauges, bien avant qu'il en fasse la découverte lui-même: « Tu es un ogre ${ }^{104}$ » (MTR 191), « Tu assouvis ta faim de chair fraîche » et « Tu me ravales au niveau du bifteck » (MTR 196). C'est encore la rupture avec Rachel qui permet à Tiffauges de se rendre compte de sa double nature, reconnaissance qui se double de la découverte de son écriture gauche:

Quand Rachel m'a quitté, j'ai pris la chose d'un cœur léger. Je continue d'ailleurs à juger cette rupture sans gravité, et même bénéfique d'un certain point de vue, parce que j'ai la conviction qu'elle ouvre la voie à de grands changements, à de grandes choses. Mais il y a un autre moi, le moi visqueux. Celui-là n'avait rien compris d'abord à cette histoire de rupture. Il ne comprend d'ailleurs jamais rien du premier coup. C'est un moi pesant, rancunier, humoral, toujours baigné de larmes et de semence, lourdement attaché à ses habitudes, à son passé. Il lui a fallu des semaines pour comprendre que Rachel ne reviendrait plus. Maintenant il a compris. Et il pleure. (MTR 208)

On remarquera que Tiffauges se tient ici un miroir en s'observant de l'extérieur et en se décrivant comme un être étranger à lui-même: le passage du « je » au « il » est significatif à cet égard. Ce Tiffauges-là, celui qui pleure, celui qui répète aussi qu'il a souvent $\mathrm{ri}^{105}$ avec Rachel, se montre sous un aspect humain. Dans la description de sa relation avec Rachel Tiffauges introduit même un brin d'humour par moments:

\footnotetext{
103 Voir aussi Gascoigne (1996, p. 207), et les remarques de Tournier lui-même au sujet de pseudonymes dans Tournier (2011, p. 109).

104 Il est à noter que Tiffauges, mégalomane, se distingue et s'individualise tout de suite en écrivant « Un Ogre ? » avec majuscule. Voir aussi les commentaires à ce sujet de Worton (1982), et Krell (1994, p. 90). 105 En effet le rire semble être banni du reste du roman.
} 
le double sens et le jeu de mots sur « comptable volant ${ }^{106}$ en référence au métier exercé par cette « Israélite » parmi sa clientèle juive en témoignent. Rachel de son côté se livre à des « plaisanterie[s] obscène[s] » (MTR 195), affirmant ainsi son air « garçonne » ${ }^{107}$ (MTR 194). Quelle différence entre ce Tiffauges et l'autre, le surhumain, le sinistre! Au début de la partie III du roman, après le passage du Rhin, le narrateur confirme le caractère «bénéfique » et même nécessaire de la rupture avec Rachel, toujours en relation avec la mégalomanie de Tiffauges:

Il avait laissé tomber derrière lui comme vêtements souillés, comme chausses éculées, comme peaux craquelées, Paris et la France, avec au premier plan Rachel [...]. Personne n'avait autant que lui la conscience de son destin, un destin rectiligne, imperturbable, inflexible qui ordonnait à ses seules fins les événements mondiaux les plus grandioses. (MTR 331)

Si Rachel lâche donc le Tiffauges sociable-lui dirait probablement « droit » — en France et annonce ainsi sa déroute, le Tiffauges sinistre, qui a effacé son double, lâche Rachel à son tour et à l'inverse en Allemagne. Lâcher dans les deux sens donc, le choix du nom de Rachel s'impose et s'explique ainsi tout naturellement à cause de son potentiel anagrammatique, l'inversion du $L$ et du $R$ permettant de réaliser celle des abandons de l'un par rapport à l'autre. ${ }^{108}$

À Rachel la première parole du roman, à Éphraïm la dernière:

- Il faut quitter la route, décida Éphraïm. Tu vas prendre à gauche par la lande, nous contournerons la colonne de chars. Sans discuter, Tiffauges obliqua vers le talus de gauche, s'enfonça dans les congères boueuses qui le bordaient, et sentit sous ses pieds le sol mou et traître de la brande. (MTR 521)

Totalement désorienté dans cette Prusse-Orientale, où il perd littéralement pied dans une terre qui lui était si ferme auparavant, Tiffauges s'en remet à Éphraïm pour le guider et l'orienter. ${ }^{109} \mathrm{Au}$ fur et à mesure que le rôle de Tiffauges devient plus important dans le monde du nazisme, il s'enfonce dans sa nature ogresque. À la fin du roman c'est l'inverse qui se produit: en s'enfonçant dans les marécages prussiens,

\footnotetext{
${ }^{106} \mathrm{Ce}$ jeu de mots semble faire allusion à plusieurs caractéristiques voire partis pris concernant le judaïsme, allant de la notion du Juif errant au penchant et à l'intérêt pour l'argent.

107 Tiffauges écrit que ce « type 'garçonne' [était] très en vogue depuis un certain roman à succès ». Il s'agit de La Garçonne de Victor Margueritte, paru en 1922.

108 Il n'est pas non plus exclu que ce jeu de mots anagrammatique « Rachel/Lâcher » corresponde aux rapports sexuels entre Rachel et Tiffauges. Elle lui reproche de faire l'amour « comme un serin »; lui parle de « l'acte sexuel insuffisamment retenu, différé » (MTR 196). David Gascoigne, 1986, p. 69, consacre quelques lignes bien intéressantes au rapport entre Rachel et Tiffauges. Si Tiffauges est un serin, Rachel est comparée par sa physique (nez aquilin) à l'aigle, ce qui serait une préfiguration de l'opposition entre l'aigle, symbole du nazisme prédataire, et les pigeons, proies inoffensives. Cette image s'inscrirait dans le thème de la domination/soumission (voir supra et Korthals Altes 1992, p. 59).

109 La désorientation de Tiffauges à la fin du roman est à double sens, bien sûr, figuré et littéral: la perte de ses lunettes prive Tiffauges de la vue, du coup il dépend de la seule ouie, ce qui est encore concrétisé par Éphraïm qui le guide par les oreilles. Pour une analyse plus approfondie du rapport entre Tiffauges et Éphraïm, voir Milne (1994, pp. 103-106).
} 
il regagne en humanité. ${ }^{110}$ C'est Éphräm qui avait ouvert les yeux de Tiffauges en lui révélant avec « une fidélité effrayante » (MTR 505) l'existence des camps d'extermination des Juifs. Le terme de « torpeur» (MTR 503) est le premier utilisé pour décrire l'état d'âme d'Éphraïm, après la description de sa physique. Il s'impose aussi pour décrire l'effet de la rencontre sur Tiffauges. La torpeur de Tiffauges ne fait que s'accroître au fur et à mesure qu'il écoute les révélations d'Éphraïm par la suite. Pour sortir Éphraïm de la «torpeur» où il était plongé, il lui faut le porteur qu'est Tiffauges, pour le soulever et relever, toujours selon ce même principe de l'inversion, par le sens et par la forme choisie pour l'exprimer. Sauvé des fours crématoires d'Auschwitz, Éphraïm suit le parcours inverse de Nestor, qui a péri dans le feu en rechargeant la chaudière du collège Saint-Christophe, et dans les deux cas Tiffauges y est pour quelque chose, volontairement pour l'un, accidentellement pour l'autre. Éphraïm qui dirigeait « une voiture à cheval » avec « vingt autres enfants [du] Rollkommando » (MTR 508) dans le camp de concentration, contre Nestor qui était à la tête des enfants du collège et qui joue aux « cavaliers et montures » (MTR 228). Tiffauges rachète la perte de Nestor, l'obèse, par le sauvetage d'Éphraïm, l'enfant $\mathrm{au}$ « corps délabré » (MTR 504) et « squelettique » (MTR 505), les deux pourraient d'ailleurs bien avoir le même âge. ${ }^{111}$ La fusion entre Tiffauges et Nestor est poursuivie par celle de Tiffauges et Éphraïm qui l'appelle Béhémoth, « cheval d'Israël » à la fin du roman.

Si l'origine juive ${ }^{112}$ des noms de Rachel et d'Éphraïm, ${ }^{113}$ qui bouclent ainsi le roman, est explicitée par le narrateur, le rapport « biblique » entre les deux ne l'est pas. Éphraïm était le petit-fils de Rachel et de Jacob et le fils de Joseph. Lors de la bénédiction d'Éphraïm et de son frère aîné Manassé par Jacob, celui-ci, aveugle, croise les bras et met la main droite sur la tête d'Éphraïm et la main gauche sur celle de Manassé, inversant ainsi le droit d'aînesse. ${ }^{114}$ Le choix du nom d'Éphraïm est donc loin d'être arbitraire, car par ces origines bibliques ce personnage incarne et réitère le grand thème de l'inversion du roman (Fig. 5).

Cette scène nous conduit aussi à cet autre tableau de deux enfants, au collège Saint-Christophe, décrit par le jeune Tiffauges: « Tenant ma main gauche dans sa main droite, Nestor écrivait et dessinait de la main gauche » (MTR 216).

\section{Nestor}

Étymologiquement le prénom de Nestor signifie « celui qui revient toujours », ${ }^{115}$ ce qui correspond tout à fait aux réapparitions fréquentes dans la vie et dans le journal de Tiffauges. Le nom de Nestor évoque aussi la sagesse liée à l'âge, la « stupéfiante

\footnotetext{
110 Sur la «pureté » et « une certaine innocence » de Tiffauges, voir Krell (1994, pp. 150-151), et pour « ses [...] goûts sadiques » pp. 186-187.

111 Le texte dit qu'Éphraïm « pouvait avoir indifféremment entre huit et quinze ans » (MTR 504).

112 Bouloumié (1988, p. 43), relève l'intérêt de Michel Tournier pour l'étymologie hébraïque.

113 Signalons au passage que la Porte d'Éphraïm est une des principales entrées de la ville de Jérusalem.

114 Jacob avait usurpé lui-même le droit d'aînesse qui appartenait à Ésaü, toujours par ce même principe de l'inversion.

115 Voir Bouloumié (1988, p. 45), Platten (1999b, pp. 105-106), et Petit (1986, pp. 235-236).
} 


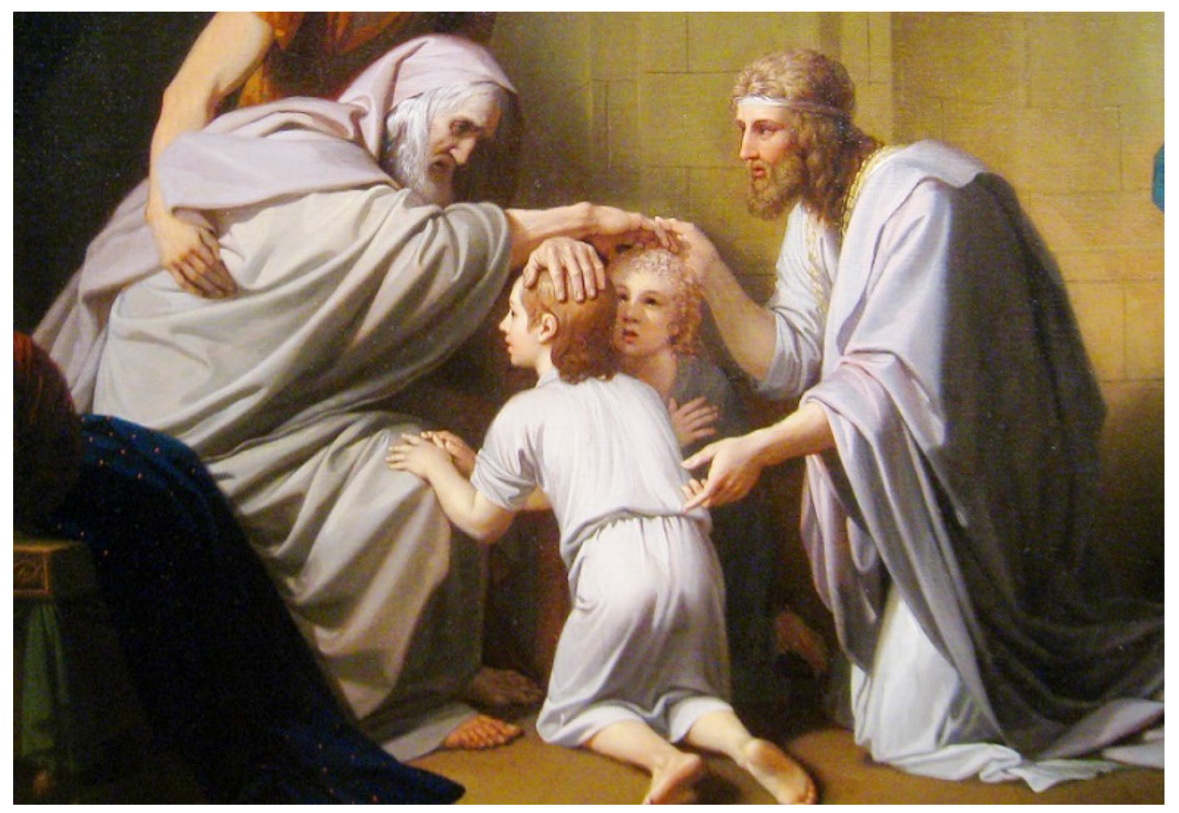

Fig. 5 Benjamin West, Jacob bénissant Éphrä̈m et Manassé. Wikimedia Commons

précocité » (MTR 205) intellectuelle du personnage tourniérien pourrait s'inscrire ainsi dans le thème omniprésent de l'inversion. Les actes et les dires de Nestor sont d'ailleurs aussi invraisemblables que ceux de Tiffauges voire plus. C'est Nestor qui lance le concept de l'Alpha et de l'Oméga ( Il faudrait réunir d'un trait alpha et oméga », MTR 221) et qui cherche à en trouver le « signe absolu » (MTR 239). La signification de ce thème d'origine religieuse ${ }^{116}$ est donnée plus loin dans le roman, à Rominten, sous forme de sacrilège: «Quant au rôle primordial du cheval dans la chasse au cerf, son sens devenait bien évident. C'était la persécution de l'Ange Phallophore par l'Ange Anal, le pourchas et la mise à mort d'Alpha par Oméga » (MTR 392). C'est la mort des trois enfants empalés, Haïo, Haro et Lothar, «percés d'oméga en alpha » (MTR 519) qui constitue la réalisation profane de la phrase de Nestor.

\footnotetext{
${ }^{116}$ L'alpha et l'oméga sont la première et la dernière lettre de l'alphabet grec. En termes bibliques elles symbolisent la toute-puissance de Dieu: «Je suis l'alpha et l'oméga, dit le Seigneur Dieu, celui qui est, qui était, et qui vient, le Tout Puissant », Apocalypse, 1:8.
} 
Sur le plan physique aussi Tiffauges lui attribue dès le début un caractère mythique en écrivant dans son journal qu' « [i]l y avait du Silène ${ }^{117}$ en lui » (MTR 207). Avec son obésité qui annonce le Tiffauges adulte, son « sexe minuscule » (MTR 240), ses lunettes, son écriture de la main gauche, sa lecture des signes, son intemporalité, Nestor est sans doute le plus important des nombreux alter ego de Tiffauges. ${ }^{118}$ Jean-Bernard Vray consacre une analyse très détaillée et convaincante au rapprochement entre Tiffauges et le personnage principal de Le désespéré de Léon Bloy, alter ego dont le nom de Caïn Marchenoir fait preuve d'autant de recherche et de jeu que ceux consentis par Tournier pour caractériser ses personnages. Vray conclut que « Tournier voit à l'œuvre chez Bloy un goût obsessionnel de l'interprétation, la fascination pour la symbolique héraldique dont il dotera aussi Tiffauges » (p. 269). Ou encore: « Le délire interprétatif rapproche singulièrement Tiffauges de Bloy [...]» (p. 273).

Si le rapprochement anagrammatique entre Nestor et Ternes est quasiment parfait quant à l'inversion syllabique, son influence est aussi presque complète sur la conduite et la vie de Tiffauges garagiste à la Porte-des-Ternes et après. Il donne à Tiffauges deux surnoms à caractère fort affectif. Le premier, diminutif, « petit Fauges » (3 fois), fait allusion à la physionomie rachitique du jeune Tiffauges dont il est le protecteur, alter ego qui peut être interprété comme faux je du point de vue de Nestor. ${ }^{119}$ Le deuxième, tout aussi affectif mais plus ambigu, « Mabel » (6 reprises), peut se comprendre comme « ma belle », bien sûr. Mais il semble faire également écho au blâme, la sanction du conseil de discipline infligée à Tiffauges, qui coûte indirectement la vie à Nestor.

À la grande surprise de Tiffauges, Nestor jouit au Collège Saint-Christophe d'un statut d'intouchable:

Son autorité sur tous les élèves était indiscutée, et les maîtres eux-mêmes paraissaient le craindre, et lui concédaient des privilèges qui m'avaient paru exorbitants au début, alors que j'ignorais qui il était. (MTR 206)

\footnotetext{
117 Référence au prologue de Gargantua où Rabelais écrit qu'Alcibiades louait Socrates en le disant semblable aux silènes. Les silènes étaient des petites boîtes décorées d'images insolites renfermant des matières précieuses. Rabelais a emprunté l'image de « silène » à son tour à Erasme, la comparaison d'une personne avec un silène étant courante parmi les humanistes. Les correspondances entre Nestor et le personnage de Rabelais sont évidentes, notamment au niveau de la goinfrerie et de la digestion. Silène est aussi le nom de la ville où Georges de Lydda, assis sur son cheval blanc, combat « un redoutable dragon qui dévore tous les animaux de la contrée et exige des habitants un tribut quotidien de deux jeunes gens tirés au sort » (https://fr.wikipedia.org/wiki/Georges_de_Lydda, consulté le 25 mai 2017). Cette histoire d'ogre tué se retrouve dans la Légende dorée de Jacques de Voragine dont la lecture est imposée à Tiffauges au Collège Saint-Christophe (MTR 224). Notons au passage que le nom de Jacques de Voragine a dû plaire à Tournier par son rapprochement avec vorace ou voracité, caractéristiques pour le thème de l'ogre.

118 Citons encore Don Juan, Weidmann, l'élan appelé Unhold, l'homme des tourbes appelé Roi des Aulnes, Saint-Christophe, Bram Johnson, le protagoniste du Piège d'or de Curwood, le fou Victor, etc. Pour Tournier, Don Juan est explicitement un ogre (Tournier 2011, p. 94). Voir aussi Korthals Altes (1992, p. 45), et Koopman-Thurlings(1993, pp. 93-105). Nestor est à son tour un alter ego de Rogier Nimier: dans Le Vent Paraclet (MTR 1417) Tournier écrit « [qu'i]l y a un peu de lui dans le Nestor du Roi des Aulnes ».

119 La traduction d'alter ego pourrait d'ailleurs être faux je d'une manière générale.
} 
Ce « prestige » et son « pouvoir » $(\mathrm{MTR} 240)$, sa « force » et son « esprit dominateur » (MTR 216) mettent Nestor sur un piédestal, trône qui est inversé dans la scène nocturne décrite dans les détails scatologiques par Tiffauges sur plusieurs pages:

Le siège de bois sombre était bizarrement juché sur une manière de podium à deux marches, véritable « trône » qui se dressait pompeusement dans le fond de la pièce. Nestor me tourna le dos et gravit ces degrés lentement, comme accomplissant déjà un acte rituel. Parvenu au pied de son trône, il fit glisser son pantalon qui tomba en tire-bouchon sur ses pieds. (MTR 239-240)

$\mathrm{Au}$ lieu de ridiculiser Nestor, cette scène a un effet contraire sur Tiffauges, qui « [s]e $\mathrm{mi}[\mathrm{t}]$ pour la première fois à l'aimer » (MTR 240). C'est précisément dans cette position avilissante qu'aux yeux de Tiffauges le prestige philosophique et même religieux voire sacré ${ }^{120}$ de Nestor se réalise pleinement: « Il se posa sur le trône et ressembla aussitôt à un sage hindou, à un bouddha méditatif et bienveillant » (MTR 240). Les deux sens contraires et, dans l'esprit du roman, sans doute complémentaires du mot trône se rejoignent dans le « plaisir royal » (MTR 241) éprouvé par Nestor à la fois au niveau de son prestige et de sa défécation. ${ }^{121}$ Or, ces deux trônes opposés si longuement décrits et si caractéristiques anagrammatiquement réunis dans le personnage de Nestor sont le reflet exact de son prénom. ${ }^{122}$ Nestor est ainsi la personnification du thème de l'Alpha et de l'Oméga introduit par lui-même, jusque dans son nom.

\section{Adolf Hitler et Hermann Goering (Göring)}

Dans son chapitre intitulé « La déchirure », ${ }^{123}$ Bouloumié (1988, p. 128) consacre des pages très intéressantes au mythe des jumeaux et à leurs noms. Pour elle « [ce mythe] finit par exprimer l'écartèlement de la conscience entre le bien et le mal. C'est le cas pour Gilles \& Jeanne où les noms historiques semblent se plier à l'intuition gémellaire de l'auteur. Les deux noms ont le même rythme binaire, la même fricative au début. Seul, le i s'oppose au a, le 1 au n. Mais Gilles est le jumeau démoniaque de Jeanne d'Arc ». Ailleurs ${ }^{124}$ nous avons également attiré l'attention sur ce titre et la combinaison de ces deux noms historiques. Qu'en est-il sur ce plan pour Le Roi des Aulnes? Est-ce que Tournier a trouvé moyen de tirer profit de manière significative (des noms) des personnes de l'Histoire devenues de ce fait même des personnages historiques dans ce roman?

\footnotetext{
120 Korthals Altes (1993, pp. 77-91) parle de « l'esthétique du grotesque qui se nourrit de l'inversion des valeurs » et constate qu' « [a]vec Tiffauges, comme son initiateur et précurseur Nestor, la défécation [...] devient un art [...] ». Gascoigne (1996, p. 76), constate que le trône pour le rituel défécatoire de Nestor est devenu « autel » pour Tiffauges adulte (MTR 341 et 343).

121 Dans une interview avec Koster (1984, p. 196), Tournier déclare: « Plus on s'enfonce dans la merde, plus on monte dans le ciel ». Voir aussi Dalmas (2005, pp. 91-109).

122 Il va sans dire que le mot trône au singulier est également un anagramme de Nestor, il suffit pour cela de remplacer l'accent circonflexe par le $-\mathrm{s}$.

123 Bouloumié (1988, p. 128).

124 Voir à ce sujet notre article écrit en collaboration avec Smith (2009, pp. 187-189).
} 
Éphraïm échappe à l'extermination des Juifs organisée et orchestrée par le régime nazi, dont le chef, Adolf Hitler, n'épargne pas non plus ses propres jeunes citoyens. Tiffauges s'en rend compte seulement à Rominten à l'occasion de l'anniversaire du Führer:

- Vous ne savez pas que le 20 [avril] c'est l'anniversaire de notre Führer? Chaque année la nation allemande lui offre en cadeau d'anniversaire toute une génération d'enfants! (MTR 401).

Tournier s'explique à ce sujet comme suit:

À mesure que j'avançais dans mes recherches, je voyais affluer des détails qui confirmaient la vocation ogresse du régime nazi. L'un des plus frappants est cette date du 19 avril à laquelle solennellement tous les petits garçons et toutes les petites filles ayant eu dix ans dans l'année - un million d'enfants au total, quel beau chiffre bien rond! - étaient incorporés, les uns dans le Jungvolk, les autres dans le Jungmädelbund. Pourquoi le 19 avril? Parce que le 20, c'était l'anniversaire d'Hitler. Le Führer prenait ainsi des airs d'Ogre Majeur, de Minotaure auquel pour son anniversaire on fait offrande de toute une génération de petits enfants. ${ }^{125}$

Dans une lettre à Volker Schlöndorff, metteur en scène du film tiré du roman, Tournier insiste pour que cette date soit ajoutée au scénario. ${ }^{126}$ Sachons que la fête des Éphraïm tombe également le 20 avril, la même date donc que l'anniversaire du plus grand ogre de tous les temps, l'ogre de Rastenburg... Si le nom d'Hitler ne présente guère des possibilités au niveau onomastique, Tournier réussit quand même à l'intégrer dans son thème de l'inversion maligne, par le biais du choix du nom d'Éphraïm. Nous pensons que la coïncidence de ces deux anniversaires est symbolique et bien plus qu'un hasard: il pourrait très bien s'agir ici d'un pied de nez, d'une grimace du judaïsme vis-à-vis de son grand exterminateur. Tournier ne fuit pas ce genre de concours de circonstances, au contraire, on pourrait même dire qu'il les recherche. Ainsi la population locale s'en prend, en vain, à Tiffauges le 20 juillet 1944, la même date de l'attentat échoué contre Hitler. Ou encore le repas rituel consumé par l'homme des tourbières appelé le Roi des Aulnes, qui serait tombé le même jour de la dernière cène, et le jour de naissance de l'assassin Weidmann, le 5 février 1908, qui coïncide avec celui de Tiffauges. ${ }^{127}$

\footnotetext{
125 Le Vent Paraclet, MTR 1384. Voir aussi Le Monde (1970).

126 Lettre datée du 28 février 1995 et reproduite dans Bouloumié (2016, p. 186).

127 Voici encore trois autres exemples des coïncidences relevées ou recherchées par Tournier:

Dans le chapitre "Inversion bénigne, inversion maligne », Bouloumié (1991, pp. 35-36) parle de la découverte de l'été 1989 par Michel Tournier (1989): « l'explosion de de la bombe atomique d'Hiroshima eut lieu un 6 août:«Imaginez la lumière, les visages transformés des hommes d'Hiroshima. » Or le 6 août est la fête de la Transfiguration du Christ. « Les corps sont rendus au soleil. Dans saint Matthieu le visage de Jésus sur le mont Thabor 'resplendit comme le soleil et ses vêtements devinrent blancs comme la lumière' (Matthieu, 17-1). Or ce sont des chrétiens qui ont envoyé la bombe à des non-chrétiens. Voilà un exemple d'inversion maligne ».

Dans la postface du même recueil, p. 394, Tournier montre encore son intérêt pour la coïncidence des anniversaires en évoquant le petit-fils de Per Christensen qui a « choisi » (!) de naître le jour même de l'anniversaire de son grand-père norvégien.
} 
L'addition des chiffres des deux anniversaires dont il est question plus haut, le 20 avril et le 20 juillet, correspond aux 40 napolas; leur multiplication concorde avec les 400 garçons de dix à dix-sept ans formés dans chaque napola. S'il est encore question ici d'un hasard, c'est du moins un hasard recherché par l'auteur, tous ces chiffres étant mentionnés explicitement dans le roman. Ce type de d'opérations de calcul, qui s'apparentent au numérique kabbalistique, a été relevé aussi par Gascoigne (1996, p. 8), qui observe que le roman se compose de 6 chapitres, 84 fragments datés répartis sur 3 sections de 36,18 et 30 fragments datés chacune. Gascoigne constate que « [c] uriously, all these groupings $-36+18+30=84-$ within the first of the six parts of the novel are themselves multiples of six, which seems unlikely, in the hands of such a self-conscious constructor of narrative as Tournier, to be a coincidence ». L'explication de l'importance du chiffre «six » est donnée dans la dernière phrase du roman, par l'image de « l'étoile à six banches », signe de judéité (MTR 521).

Le cas de Goering est plutôt spécial aussi. La rencontre de Tiffauges avec Goering ${ }^{128}$ se fait suite à la « mésaventure » (MTR 368) de celui-là, à savoir la charge de deux troupeaux d'aurochs à laquelle il réussit à s'échapper de justesse. Cet incident lui arrive au moment précis où il passe en mémoire son passé récent: sa «migration vers le levant », «l'affaire Martine et la guerre qu'elle avait provoquée » (!), « la survenue de l'Unhold et de l'homme des tourbières » et le soupçon « qu'il rejoindrait peut-être finalement la nuit immémoriale du Roi des Aulnes » (MTR 368). Réflexions sérieuses s'il en est du point de vue de Tiffauges, et qui contrastent avec la moquerie dont il est l'objet de la part du « deuxième personnage du Reich » qui hurle de rire en se tapant sur les cuisses pendant le compte rendu (en allemand) de cet incident par Tiffauges. Cette moquerie se porte ensuite sur ses lunettes « et Tiffauges découvrit pour la première fois l'une des marottes des maîtres du III ${ }^{\mathrm{e}}$ Reich, cette haine de l'homme à lunettes, incarnant pour eux l'intelligence,

\section{Footnote 127 (continued)}

Dans une interview Tournier (2011, p. 201) déclare: «J'ai un dictionnaire encyclopédique dans lequel figurent non seulement l'année de mort et de naissance des hommes célèbres, mais la date au jour près. Je me suis aperçu, et je me demande qui le sait, que Shakespeare et Cervantès sont morts le même jour, le 23 avril 1616. C'est énorme! Le plus grand écrivain espagnol et le plus grand dramaturge anglais sont morts le même jour, la même année !».

Voir aussi nos remarques supra sur l'intérêt que porte Tournier aux coïncidences.

Rasson (2013) relève la passivité de Tiffauges et constate que « le fait [que], de simple prisonnier de guerre, [il] se mue en dignitaire nazi ne relève pas de son ambition, mais d'un concours de circonstances ». Plus loin Rasson précise que « c'est par un concours de circonstances [que Tiffauges] en vient, petit à petit, à assumer des responsabilités dans un appareil nazi qui, il est vrai, exerce un attrait particulier sur lui dans la mesure où il offre une soupape à ses pulsions sexuelles ». La coïncidence entre les attentats ratés commis le 20 juillet 1944 contre Tiffauges et Hitler pousse Rasson à se demander si celuilà n'est pas l'alter ego de celui-ci.

128 Avec la rencontre physique directe entre un personnage fictif et une personne historique tout auteur se lance dans une affaire hasardeuse, l'invraisemblance pouvant facilement dominer l'interprétation par le lecteur. Rien de tel dans Le Roi des Aulnes, et c'est sans doute une autre preuve de la qualité littéraire de l'œuvre de Tournier: une fois le personnage de Tiffauges connu et « accepté » et celui de Goebbels reconnu « conforme » par le lecteur, qui, certes, a été préalablement conditionné et manipulé par l'auteur, leur rencontre semble être découlée d'une logique tout à fait plausible. Voir aussi Korthals Altes (1992, pp. 180-181) pour les effets de l'apparition de personnages historiques dans le roman mythique. 
l'étude, la spéculation, bref le Juif » (MTR 371). ${ }^{129}$ Goering, qui fait penser Tiffauges à Nestor et par sa corpulence et par son attrait de la scatologie (MTR 380), se fait accompagner d'un autre ogre, le lion appelé « Buby ». Outre les différentes descriptions de Goering, qui sont presque toutes teintées d'ironie, le résumé fait par le narrateur de l'activité vénale du Reichsmarschall prend le format d'une tragédie classique:

Forcer un cerf, le tuer, l'émasculer, manger sa chair, lui voler ses bois pour s'en glorifier comme d'un trophée, telle était donc la geste en cinq actes de l'ogre de Rominten, sacrificateur officiel de l'Ange Phallophore. Il en existait un sixième, plus fondamental encore, que Tiffauges devait découvrir quelques mois plus tard. ${ }^{130}$ (MTR 378)

Tout dans cette quatrième partie du roman intitulé L'Ogre de Rominten tourne ainsi autour de la nature ogresse de la personne historique de Goering. Or, nous pensons que Tournier n'aurait pas opté de la même manière et avec la même insistance pour la présence (im)pertinente, proéminente et dévoratrice de ce grand ogre dans le roman si les quatre premières lettres du patronyme de celui-ci, Goering, n'avaient pas été précisément les mêmes que celles du mot ogre. Goering incarne non seulement le thème de l'ogre, il l'englobe anagrammatiquement jusque dans son nom. ${ }^{131}$

\section{Conclusion}

Le Roi des Aulnes est un roman «musical » et un roman d'aventures, empreint de signes, roman qui reflète un monde à la fois historique et mythologique, ${ }^{132}$ monde également empreint de signes et de symboles. Limiter son attention en tant que lecteur aux seules difficultés d'interprétation rencontrées par le protagoniste, qui, en tant que diariste-narrateur, œuvre certes dans ce sens par son caractère dévorateur, c'est passer outre au travail d'interprétation, de conception, de fiction et

\footnotetext{
129 Contrairement à la scène avec Blättchen plus tard dans le roman, Tiffauges ne réagit pas à cette provocation. Il n'en tire pas non plus aucune leçon quant à la vraie nature du Nazisme et semble ignorer totalement ce signe qui est pourtant à ne pas manquer. Le port des lunettes est aussi la raison du mépris du professeur Otto Essig de la part de Goering, mépris qui tourne en colère quand celui-ci comprend que la myopie d'Essig est la cause de l'abattement du plus beau cerf de Rominten.

130 Pour ce sixième acte il s'agit, bien sûr, de la révélation de l'existence des camps d'extermination par Éphraïm.

131 Tournier (2011, p. 62) fait le rapprochement direct entre ogre et Goering (épelé tel quel): « Je voyais passer l'ogre Goering, le gros lourd rigolard qui se voulait populaire » (on remarquera aussi la concordance répétée quatre fois du son $[\mathrm{g}]$ ). Tournier savait-il que le SS qui dirigeait la récupération des affaires et le vol des richesses des Juifs, surnommé le « comptable d'Auschwitz », le responsable du Canada, s'appelait Oskar Groening ? Ayant repris après la Seconde Guerre mondiale une vie normale en Allemagne, où il a occupé la fonction de juge industriel et commercial, Groening a été condamné à quatre ans de prison lors d'un procès commencé seulement le 20 avril (!) 2015 devant le tribunal régional de Lüneburg.
}

132 Dans Le Vent Paraclet Tournier écrit: « Roman apparemment* historique Les Météores élève l'événement à la puissance mythologique en effectuant une déduction romanesque de l'Histoire » (MTR 1404). La même chose pourrait se dire pour Le Roi des Aulnes. 
d'écriture consenti par l'auteur au niveau des signes historiques et des symboles mythologiques, pour le fond et pour la forme. En d'autres termes, bien que l'auteur ait conçu Le Roi des Aulnes d'une telle manière que le lecteur aurait tendance à se laisser manger par l'ogre Tiffauges, qui transpose ainsi sa nature ogresse même au niveau de la lecture en tentant de s'emparer de l'interprétation, le roman présente une richesse sémiotique qui mérite encore bien d'autres lectures. Une fois admis que la lecture de la lecture des signes selon Tiffauges n'est pas une fin en soi car limitative, la nôtre, effectuée à rebours comme pour répondre au principe d'écriture de Tournier et au thème de l'inversion, a permis de confirmer une application particulière et de découvrir une recherche parfois insolite de la part de l'auteur au niveau de l'onomastique. Avec l'interférence et l'interdépendance, apparentes ou moins évidentes, entre personnages, historiques ou non, lieux, événements et leurs noms ou appellations, Tournier, en jouant sur les mots et en anagrammatisant les lettres, inscrit ce jeu formel dans le thème de l'inversion et soumet au lecteur averti autant de signes qu'il invite à découvrir et dont il convient d'intégrer la multiple interprétation dans la genèse et la signification globale du roman.

Open Access This article is distributed under the terms of the Creative Commons Attribution 4.0 International License (http://creativecommons.org/licenses/by/4.0/), which permits unrestricted use, distribution, and reproduction in any medium, provided you give appropriate credit to the original author(s) and the source, provide a link to the Creative Commons license, and indicate if changes were made.

\section{Références}

Alessandrelli, S. (2013). Michel Tournier: De l'ironie à l'humour, du roman au texte bref. Modernité de Michel Tournier (pp. 39-53). Rennes: Presses universitaires de Rennes. (Coll. Interférences).

Bataille, G. (1965). Le Procès de Gilles de Rais. Paris: J.-J. Pauvert.

Bergholz, R. (2010). A Simonidean tale. Commemoration and coming to terms with the past: Michel Tournier's Le Roi Des Aulnes. Journal of the Australasian Universities Language and Literature Association. https://doi.org/10.1179/000127910804775478.

Bevan, D. G. (1986). Michel Tournier. Amsterdam: Rodopi.

Bouloumié, A. (1988). Michel Tournier, Le roman mythologique. Paris: Librairie José Corti.

Bouloumié, A. (1989). Rencontre avec Michel Tournier. Europe, pp. 147-157.

Bouloumié, A. (1991). Inversion bénigne, inversion maligne. Dans Images et signes de Michel Tournier, Actes du colloque du Centre Culturel International de Cerisy-la-Salle, sous la direction de Arlette Bouloumié et Maurice de Gandillac. Paris: Gallimard.

Bouloumié, A. (1993). La séduction de la réécriture chez Michel Tournier: Réminiscence, ambivalence, jeux d'échos et de miroir. Revue des Sciences Humaines, 232, 9-20.

Bouloumié, A. (2013). Transfiguration de la réalité par le mythe dans quelques contes de Michel Tournier. Tangence, 101, 77-92.

Bouloumié, A. (2014). Germanic variations on the theme of Phoria in 'The Erl-King'. D. M. Worton (Ed.), Michel Tournier. New York: Routledge.

Bouloumié, A. (2016). Modernité de Michel Tournier. Rennes: Presses universitaires de Rennes. (Coll. Interférences).

Dalmas, F. (2005). L'alchimie de l'excrément comme alchimie de l'Homme dans Le Roi des Aulnes de Michel Tournier. French Forum, 30(3), 91-109. https://doi.org/10.1353/frf.2006.0002.

Davis, C. (1991). Les interprétations. Dans Images et signes de Michel Tournier, Actes du colloque du Centre Culturel International de Cerisy-la-Salle, sous la direction de Arlette Bouloumié et Maurice de Gandillac (pp. 191-206). Paris: Gallimard.

Gascoigne, D. (1996). Michel Tournier. New directions in European writing. Oxford: Berg.

Le Magazine littéraire, Brochier, J.-J. (1981). «Qu'est-ce que la littérature: un entretien avec Michel Tournier »(Vol. 179). 
Jiménez, P. P. (2016). Portrait de lecteur en ogre. Sur un passage de 'Le Roi des Aulnes'. Cédille revista des estudios franceses, 12, 327-337.

Klettke, C. (1993). La musique dans l'esthétique de la 'mythécriture'de Michel Tournier: une musique textuelle de la séduction. Revue des Sciences Humaines, 232, 47-66.

Koopman-Thurlings, M. (1991). De la forme et du fond: le redoublement discursif. Dans Images et signes de Michel Tournier, Actes du colloque du Centre Culturel International de Cerisy-la-Salle, sous la direction de Arlette Bouloumié et Maurice de Gandillac (pp. 279-293). Paris: Gallimard.

Koopman-Thurlings, M. (1993). Narcisse et son double. Revue des Sciences Humaines, 232, 93-105.

Koopman-Thurlings, M. (1995). Vers un autre fantastique, Étude de l'affabulation dans l'œuvre de Michel Tournier. Amsterdam: Rodopi. (Faux titre).

Korthals Altes, L. (1992). Le salut par la fiction?, Sens, valeurs et narrativité dans 'Le Roi des Aulnes' de Michel Tournier. Amsterdam: Rodopi.

Korthals Altes, L. (1993). Du grotesque dans l'œuvre de Michel Tournier. Revue des Sciences Humaines, 232, 77-91.

Koster, S. (1995). Michel Tournier. Paris: Éditions Julliard.

Krell, J. F. (1994). Tournier élémentaire. West Lafayette, IN: Purdue University Press.

Lehtovuori, E. (1995). Les Voies de Narcisse ou le Problème du Miroir chez Michel Tournier. Helsinki: Suomalainen Tiedeakatemia.

Le Monde. (1970). De Robinson à l'ogre: un créateur de mythes. Propos recueillis par Jean-Louis de Rambures, 24 novembre.

Magnan, J.-M. (1991). La création critique ou l'avocat du diable. Dans Images et signes de Michel Tournier, Actes du colloque du Centre Culturel International de Cerisy-la-Salle, sous la direction de Arlette Bouloumié et Maurice de Gandillac (pp. 207-224). Paris: Gallimard.

Mercié, J.-L. (1991). L'ogre de Gif (Tournier photographe). Dans Images et signes de Michel Tournier, Actes du colloque du Centre Culturel International de Cerisy-la-Salle, sous la direction de Arlette Bouloumié et Maurice de Gandillac (pp. 252-254). Paris: Gallimard.

Miguet, T. (1991). L'argument ontologique comme 'monstrance'. Dans Images et signes de Michel Tournier, Actes du colloque du Centre Culturel International de Cerisy-la-Salle, sous la direction de Arlette Bouloumié et Maurice de Gandillac (pp. 179-182). Paris: Gallimard.

Milne, L. (1994). L'Evangile selon Michel: la Trinité initiatique dans l'œuvre de Tournier (Vol. 82). Amsterdam: Rodopi. (Faux titre).

Petit, S. (1986). Fugal structure, Nestorianism, and St. Christopher in Michel Tournier's "Le Roi des aulnes". Fiction, 19(3), 232-245.

Platten, D. (1991). Terms of reference: Michel Tournier's ‘Le Roi des Aulnes'. Journal of European Studies, 1, 1991, 281-302. https://doi.org/10.1177/004724419102100403.

Platten, D. (1999a). From riches to rags: Tournier and the World outside the Text: A review article. The Modern Language Review, 94(3), 673-679.

Platten, D. (1999b). Michel Tournier and the metaphor of fiction. Liverpool: Liverpool University Press.

Purdy, A. (1993). Séduction et simulation: l'empire des signes dans Le Roi des Aulnes. Revue des Sciences Humaines, 232, 21-33.

Queneau, R. (1980). Courir les rues - Battre la campagne - Fendre les flots (Vol. 150). Paris: Gallimard. (Collection « Poésie »).

Rasson, L. (2013). De Tiffauges à Aue. D. M. Dambre (Ed.), Mémoires occupées (pp. 119-128). Paris: Presses Sorbonne Nouvelle. http://www.openedition.org/6540.

Redfern, W. D. (1985). Approximating man: Michel Tournier and play in language. Modern Language Review, 80(2), 304-319.

Redfern, W. D. (1996). Michel Tournier: 'Le Coq de bruyère'. Madison: Fairleigh Dickinson University Press.

Sankey, M. (1991). La parodie: l'exemple du 'Roi des Aunes'. Dans Images et signes de Michel Tournier, Actes du colloque du Centre Culturel International de Cerisy-la-Salle, sous la direction de Arlette Bouloumié et Maurice de Gandillac (pp. 325-340). Paris: Gallimard.

Smith, P. J., \& Van der Toorn, N. (2009). Tournier bricoleur: écrire Gilles \& Jeanne. Dans Réécrire la Renaissance, de Marcel Proust à Michel Tournier (Vol. 330, pp. 171-189). Amsterdam: Rodopi. (Faux titre).

Tournier, M. (1980). Le Coq de bruyère (Vol. 1229)., Entretiens avec Michel Martin-Roland Paris: Gallimard. (Collection Folio).

Tournier, M. (1989). Six août, jour de splendeur et de terreur, La Transfiguration, Hiroshima. Le Figaro, 6 août. 
Tournier, M. (1996). Le Pied de la lettre (version augmentée) (Vol. 2881). Paris: Gallimard. (Collection Folio).

Tournier, M. (2011). Je m'avance masqué(Vol. 5685)., Entretiens avec Michel Martin-Roland Paris: Gallimard. (Collection Folio).

Tournier, M. (2015). Lettres parlées à son ami allemand Hellmut Waller. Paris: Gallimard.

Tournier, M. (2017). Romans suivis de Le vent Paraclet. Paris: Éditions Gallimard, Bibliothèque de la Pléiade.

Tumanov, V. (1999). John and Abel in Michel Tournier's 'Le Roi des Aunes'. Romanic Review, 90(3), $417-434$.

Vray, J.-B. (1997). Michel Tournier et l'écriture seconde. Lyon: Presses universitaires de Lyon.

Worton, M. (1982). Myth-Reference in 'Le Roi des Aulnes'. Stanford French Review, 299-310. http:// discovery.ucl.ac.uk/id/eprint/35902.

Worton, M. (1986). Intertextuality: To inter textuality or to resurrect it. In D. Kelley \& I. Llasera (Eds.), Crossreferences: Modern French theory and practice of criticism, Society for French Studies (Vol. 21). 